ITP-UU-08/38, SPIN-08/29

\title{
The graviton one-loop effective action in cosmological space-times with constant deceleration
}

\author{
T. M. Janssen*, T. Prokopec ${ }^{\ddagger}$ \\ Institute for Theoretical Physics 83 Spinoza Institute, Utrecht University, \\ Leuvenlaan 4, Postbus 80.195, 3508 TD Utrecht, THE NETHERLANDS
}

\begin{abstract}
We consider the quantum Friedmann equations which include one-loop vacuum fluctuations due to gravitons and scalar field matter in a FLRW background with constant $\epsilon=-\dot{H} / H^{2}$. After several field redefinitions, to remove the mixing between the gravitational and matter degrees of freedom, we can construct the one loop correction to the Friedmann equations. Due to cosmological particle creation, the propagators needed in such a calculation are typically infrared divergent. In this paper we construct the graviton and matter propagators, making use of the recent construction of the infrared finite scalar propagators calculated on a compact spatial manifold in [1]. The resulting correction to the Friedman equations is suppressed with respect to the tree level contribution by a factor of $H^{2} / m_{p}^{2}$ and shows no secular growth.
\end{abstract}

PACS numbers: 04.30.-m, 04.62.+v, 98.80.Cq

*T.M.Janssen@uu.nl, \ $\quad \ddagger$ T.Prokopec@uu.nl 


\section{Introduction}

Since the classic work of 't Hooft and Veltman on quantum gravity on flat space-time [2], many authors have studied the quantum behavior of gravitons. In particular, because of the potential relevance for inflationary cosmology, the quantum behavior of gravitons on a (locally) de Sitter background has been a widely studied subject over the past years [3, 4, 5, 6. 17, 8, 10, 11, 12, 13, 14, 15, 16, 17, 18, 19, 20, 21, 22, 23, 24, 25, 26, 27, 28, 29]. Of course de Sitter space is interesting for its relevance for inflationary cosmology. Therefore there are several works that study the potential influence of quantum behavior on inflationary observables [30, 31, 32, 33]. Another line of research deals with the back-reaction of gravitational waves on the background space-time [16, 17, 19, 20]. However of more interest for the present work is the one loop back-reaction by virtual gravitons on a de Sitter background, which has been calculated by several authors, using different techniques [13, 28, 29]. Since it is not clear whether in these works exactly the same quantity is calculated and the renormalization schemes differ, the numerical coefficients differ. However the main result is the same: one loop graviton contributions to the expectation value of the energy momentum tensor result in a finite, time independent shift of the effective cosmological constant. Since the contribution can always be absorbed in a counterterm [28], the exact numerical coefficient coming from such a calculation has no physical meaning.

The goal of this paper is to go beyond the works mentioned above and calculate the one loop effective action induced by gravitons in a more general background space-time using dimensional regularization. The geometry we consider is a Friedmann-Lemaitre-RoberstonWalker (FLRW) geometry with Hubble parameter $H=\frac{\dot{a}}{a}$ and the additional constraint that

$$
\epsilon \equiv-\frac{\dot{H}}{H^{2}}
$$

is a constant. If the universe contains only one type of matter, e.g. dust, radiation or a cosmological constant, this constraint is satisfied. In particular in matter era we have that $\epsilon=3 / 2$, in radiation era $\epsilon=2$ and de Sitter space corresponds to the limit $\epsilon \rightarrow 0$. One immediate problem with working in such a space-time, instead of de Sitter, is that for consistency of the Einstein equations the addition of matter fields is unavoidable. Whereas in de Sitter space, the only relevant metric fluctuations are the tensor modes (gravitational waves), in a more general setting one has to take the mixing of gravitational and matter degrees of freedom into account [34, 35, 23, 24, 25, 26].

Naively one might think that any back-reaction will be insignificant, since it will in a cosmological setting typically be suppressed by $H^{2} / m_{p}^{2}$, with $m_{p}$ the Planck mass. The reason 
that this is not necessarily the case has to do with a second complication. This complication is that gravitons on a cosmological background space-time behave, apart from the tensorial structure, somewhat similar to massless scalars, with a certain amount of coupling to the Ricci scalar. Such a scalar field however is long known to posses problems in the infrared 36, 37, for a wide class of cosmological backgrounds. What happens is that due to cosmological particle production correlations of the Bunch-Davies vacuum grow too fast, causing the propagator to diverge in the infrared. Such a divergence should of course be regulated and in a recent work [1] the massless scalar propagator on any constant $\epsilon$ cosmological space-time was constructed, by assuming that the universe is described by a spatially compact manifold. Effectively this simply implies that any momentum integral has an infrared cut-off at some scale $k_{0}$. In this work, we shall use the propagators constructed in [1] to obtain an infrared finite answer.

While the answers obtained in this way are infrared finite, particle production is of course a physical phenomenon. Long range correlations will therefore still be enhanced as time goes on. Therefore initially small quantum fluctuations, might - at least in principle - grow significantly in time. The contribution of these fluctuations to the stress energy tensor might therefore also grow in time, leading to a potentially significant back-reaction via the Einstein equations on the background space-time.

It is precisely this effect, that has led to several papers concerning quantum contributions to the stress energy tensor in the case of de Sitter space-time [6, 10, 8, 23, 24, 25, 38. In these works it is found that at two loop order, the backreaction due to gravitons might become significantly, while for scalars this might happen at three loop order. Such behavior, if indeed physically viable, has profound implications for the cosmological constant problem (see e.g. Ref. [39] for a review). In this paper we will not consider these higher loop effects, but we shall perform a one loop calculation, but in a more general space-time.

In section 2 we briefly review our background geometry. In section 3 we briefly summarize the construction of the propagator as given in [1]. In section 4 we apply these results to calculate the relevant kinetic operators and propagators in a model with both matter and gravitational degrees of freedom. In section 4.2 we calculate the one-loop effective action contribution to the quantum corrected Friedmann equations and renormalize the theory. We discuss our results in section 4.3 and we conclude in section 5 . 


\section{Geometry}

The background space-time we consider is is the Friedmann-Lemaittre-Robertson-Walker (FLRW) geometry in conformal coordinates

$$
g_{\mu \nu}=a^{2} \eta_{\mu \nu} \quad ; \quad \eta_{\mu \nu}=\operatorname{diag}(-1,1,1,1),
$$

with the additional constraint,

$$
\epsilon \equiv-\frac{\dot{H}}{H^{2}}=\mathrm{constant} \quad ; \quad H \equiv \frac{\dot{a}}{a}
$$

Here a dot indicates a derivative with respect to cosmological time $t$, related to the conformal time $\eta$ by $d t=a d \eta$. The FLRW geometry obeys the Friedmann equations

$$
\frac{3 H^{2}}{\kappa}-\frac{1}{2} \rho_{M}=0 \quad ; \quad-\frac{2 \dot{H}}{\kappa}-\frac{1}{2}\left(\rho_{M}+p_{M}\right)=0, \quad \kappa=16 \pi G_{N},
$$

with $G_{N}$ being the Newton constant, $\rho_{M}$ and $p_{M}$ are the energy density and pressure due to matter. If one writes

$$
p_{M}=w_{M} \rho_{M}
$$

one immediately finds that (3) implies that $w_{M}$ is constant. One can solve (4) for $a$ to find

$$
\begin{array}{ccc}
a(\eta)=\left((\epsilon-1) H_{0} \eta\right)^{-1 /(1-\epsilon)} & ; \quad \epsilon=\frac{3}{2}\left(1+w_{M}\right) \\
H=H_{0}\left((\epsilon-1) H_{0} \eta\right)^{\epsilon /(1-\epsilon)}=H_{0} a^{-\epsilon} & ; \quad a \eta=-\frac{1}{1-\epsilon} \frac{1}{H}
\end{array}
$$

Notice that if $\epsilon<1, \eta$ is negative and the expansion of the universe is accelerating. If $\epsilon>1$, $\eta$ is positive and the expansion is decelerating. $H_{0}$ is chosen such that the $\epsilon \rightarrow 0$ limit of $H$ corresponds to the one given in [22]. Given two points $x$ and $\tilde{x}$, the following distance function will prove to be useful

$$
y(x ; \tilde{x})=\frac{\Delta x^{2}(x ; \tilde{x})}{\eta \tilde{\eta}}=\frac{1}{\eta \tilde{\eta}}\left(-(|\eta-\tilde{\eta}|-\imath \varepsilon)^{2}+\|\vec{x}-\overrightarrow{\tilde{x}}\|^{2}\right) .
$$

Here the infinitesimal $\varepsilon>0$ refers to the Feynman (time-ordered) pole prescription. In the following we shall omit the explicit argument of $y(x ; \tilde{x})$, since this will be clear from the context. In de Sitter space $y$ is related to the geodesic distance $l$ as $y=4 \sin ^{2}\left(\frac{1}{2} H l\right)$. If $y<0$, points $\tilde{x}$ are timelike related to $x$, and if $y>0$, they are spacelike related. We define the antipodal point $\bar{x}$ of $x$ by the map $\eta \rightarrow-\eta$ [40]. Notice that, since as long as $\epsilon$ is 


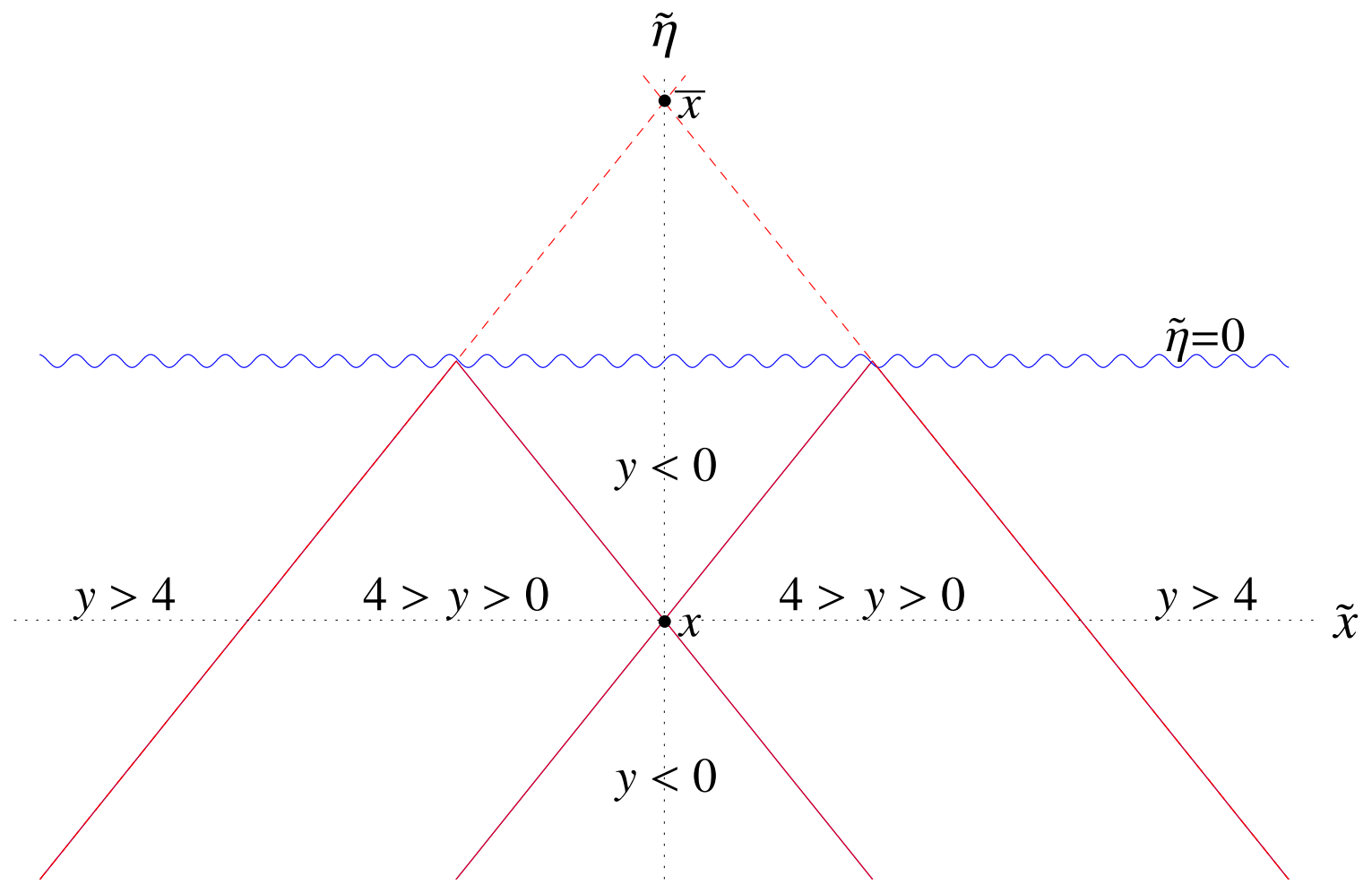

Figure 1: The causal structure in the conformal coordinates (2). The plot assumes $\epsilon<1$, so the coordinates cover only the region $\eta<0$. The wavy line at $\eta=0$ indicates future infinity. The lightcone of the point $x$ is given by $y=0$. If $y=4$, the point $\tilde{x}$ lies on the light cone of an unobservable image charge at the antipodal point $\bar{x}$. 
constant, $\eta$ is either positive or negative, this point is not covered by our coordinate patch. If $y=4, \tilde{x}$ lies on the lightcone of an (unobservable) image charge at the antipodal point $\bar{x}$, see figure 1 .

The Levi-Cività connection is,

$$
\Gamma_{\mu \nu}^{\alpha}=\frac{a^{\prime}}{a}\left[\delta_{\mu}^{\alpha} \delta_{\nu}^{0}+\delta_{\nu}^{\alpha} \delta_{\mu}^{0}+\delta_{0}^{\alpha} \eta_{\mu \nu}\right]
$$

while the curvature tensors are given by

$$
\begin{aligned}
R_{\mu \beta \nu}^{\alpha}= & \left(\frac{a^{\prime \prime}}{a}-2\left(\frac{a^{\prime}}{a}\right)^{2}\right)\left(\delta_{\nu}^{\alpha} \delta_{\mu}^{0} \delta_{\beta}^{0}-\delta_{0}^{\alpha} \delta_{\nu}^{0} \eta_{\mu \beta}-\delta_{\beta}^{\alpha} \delta_{\nu}^{0} \delta_{\mu}^{0}+\delta_{\beta}^{0} \delta_{0}^{\alpha} \eta_{\mu \nu}\right) \\
& -\left(\frac{a^{\prime}}{a}\right)^{2}\left(\delta_{\nu}^{\alpha} \eta_{\mu \beta}-\delta_{\beta}^{\alpha} \eta_{\mu \nu}\right) \\
R_{\mu \nu}= & \left(\frac{a^{\prime \prime}}{a}-2\left(\frac{a^{\prime}}{a}\right)^{2}\right)\left(\eta_{\mu \nu}-(D-2) \delta_{\mu}^{0} \delta_{\nu}^{0}\right)+\left(\frac{a^{\prime}}{a}\right)^{2}(D-1) \eta_{\mu \nu} \\
R= & \left(\frac{a^{\prime \prime}}{a^{3}}-2\left(\frac{a^{\prime}}{a^{2}}\right)^{2}\right) 2(D-1)+\left(\frac{a^{\prime}}{a^{2}}\right)^{2} D(D-1),
\end{aligned}
$$

where $a^{\prime}=d a / d \eta$ and $D$ denotes the number of space-time dimensions.

\section{Scalar Propagator}

Since it will turn out that the graviton propagator can be expressed in terms of massless scalar propagators with different amount of conformal coupling, we need to find a solution for the following scalar Klein-Gordon equation

$$
\sqrt{-g}(\square-\xi R) \imath \Delta(x ; \tilde{x})=\imath \delta^{D}(x-\tilde{x})
$$

where $\xi$ is a dimensionless scalar field coupling to the Ricci curvature scalar, and $\square$ denotes the d'Alembertian, which reads when acting on a scalar

$$
\sqrt{-g} \square \phi=\partial_{\mu} \sqrt{-g} g^{\mu \nu} \partial_{\nu} \phi
$$

If $\epsilon$ is constant we can rewrite this equation as follows

$$
\left[\eta^{\mu \nu} \partial_{\mu} \partial_{\nu}+\frac{1}{\eta^{2}}\left(\nu^{2}-\frac{1}{4}\right)\right]\left[(a \tilde{a})^{\frac{D}{2}-1} \imath \Delta(x ; \tilde{x})\right]=\imath \delta^{D}(x-\tilde{x})
$$

where $\tilde{a}=a(\tilde{x})$ and 


$$
\nu^{2}=\left(\frac{D-1}{2}\right)^{2}-(1-\epsilon)^{-2}\left((D-1)(D-2 \epsilon) \xi-\frac{1}{2}(D-1)(D-2) \epsilon+\frac{D}{4}(D-2) \epsilon^{2}\right) .
$$

The properties of such a scalar field propagator on a de Sitter background $(\epsilon=0$ in our notation) have been studied in the past [40, 41, 42].

This propagator in a general constant $\epsilon$ space is given in [1], by integrating over the mode functions and we simply quote the result here. The massless scalar propagator is infrared divergent if the index $\nu$ (which is defined in (13) is less then $(D-1) / 2$. Therefore we work on a compact spatial manifold, with comoving radius $k_{0}^{-1}$. In this case, the integral over the mode functions becomes a discrete sum. However, if $k_{0}$ is small enough, it is generally valid to approximate this sum by an integral, with an infrared cutoff. This approach has passed sever consistency checks on de Sitter space[6, 43, 44]. In the final propagator we can then recognize two different types of contributions: one coming from the 'infinite volume' integral (thus the integral, without an infrared cut-off), and one from the subtraction of the lower range of the integral.

$$
i \Delta(x ; \tilde{x})=i \Delta_{\infty}(x ; \tilde{x})+\sum_{N=0}^{\infty} \delta i \Delta_{N}(x ; \tilde{x})+\sum_{N=0}^{\infty} \delta i \Delta^{N}(x ; \tilde{x}) .
$$

Here the infinite volume propagator is

$i \Delta_{\infty}(x ; \tilde{x})=\frac{\left[(1-\epsilon)^{2} H \tilde{H}\right]^{\frac{D}{2}-1}}{(4 \pi)^{\frac{D}{2}}} \frac{\Gamma\left(\frac{D-1}{2}+\nu\right) \Gamma\left(\frac{D-1}{2}-\nu\right)}{\Gamma\left(\frac{D}{2}\right)}{ }_{2} F_{1}\left(\frac{D-1}{2}+\nu, \frac{D-1}{2}-\nu ; \frac{D}{2} ; 1-\frac{y}{4}\right)$

and the correction terms due to the infrared divergence are

$$
\begin{aligned}
\delta i \Delta_{N}(x ; \tilde{x})=-\frac{\left(H \tilde{H}(1-\epsilon)^{2}\right)^{\frac{D}{2}-1}}{(4 \pi)^{\frac{D}{2}}} \frac{2 z_{0}^{2 N+(D-1)-2 \nu}}{2 N+(D-1)-2 \nu} \\
\times \frac{\Gamma(2 \nu) \Gamma(\nu)}{\Gamma\left(\frac{1}{2}+\nu\right) \Gamma\left(\frac{D-1}{2}\right)} \sum_{k=0}^{N} \sum_{\ell=0}^{N-k} a_{k \ell}\left(\frac{r}{\tilde{\eta}}\right)^{2 k}\left(\frac{\eta}{\tilde{\eta}}\right)^{2 \ell-N} \\
\delta i \Delta^{N}(x ; \tilde{x})=\left.\delta i \Delta_{N}(x ; \tilde{x})\right|_{\nu \leftrightarrow-\nu},
\end{aligned}
$$

where $z_{0}=k_{0}|\eta|$ and

$$
a_{k \ell}=\left(-\frac{1}{4}\right)^{N} \frac{1}{k ! \ell !(N-k-\ell) !} \frac{\Gamma\left(\frac{D-1}{2}\right) \Gamma^{2}(1-\nu)}{\Gamma\left(k+\frac{D-1}{2}\right) \Gamma(\ell+1-\nu) \Gamma(N-k-\ell+1-\nu)} .
$$


Notice that in an accelerating space-time $(\epsilon<1) \eta$ approaches zero at late times, while in a decelerating space-time $(\epsilon>1), \eta$ approaches infinity. This is an interesting observation, since it implies that in an accelerating universe, $i \Delta_{N}$ contains terms that grow in time if $\nu<\frac{D-1}{2}$. In a decelerating universe both $i \Delta_{N}$ and $i \Delta^{N}$ can contain growing terms. It is precisely these growing terms that can lead to a secular growth of quantum effects, described in the introduction. There are also other regularizations possible for the infrared. For example in [45] the infrared is regulated by matching an infrared finite space-time to the space-time under consideration. This ensures that no infrared divergences can occur. Results calculated using this regularization are similar in accelerating space-times, but show differences in a decelerating space-time.

\section{The gravitational and matter propagators}

For our model we shall consider the action of gravity plus a scalar field $\hat{\phi}=\hat{\phi}(x)$ with an arbitrary potential $V(\hat{\phi})$

$$
S=\int \sqrt{-\hat{g}}\left(\frac{\hat{R}-(D-2) \Lambda}{\kappa}-\frac{1}{2}(\partial \hat{\phi})^{2}-V(\hat{\phi})\right),
$$

where $\kappa=16 \pi G_{N}=16 \pi / m_{\mathrm{P}}^{2}$ denotes the (rescaled) Newton constant, $m_{\mathrm{P}} \simeq 1.2 \times 10^{19} \mathrm{GeV}$ is the Planck mass, $\Lambda$ denotes the cosmological constant, $D$ is the number of space-time dimensions and $(\partial \hat{\phi})^{2}=\hat{g}^{\mu \nu}\left(\partial_{\mu} \hat{\phi}\right)\left(\partial_{\nu} \hat{\phi}\right)$. By an appropriate choice of the potential $V$, such a model can mimic any mixture of fluids which are relevant for the evolution of the univese. We consider a conformally flat FLRW background and split the fields in a background contribution and a quantum contribution [7] [26]

$$
\begin{aligned}
\hat{g}_{\mu \nu} & =g_{\mu \nu}(\eta)+\delta g_{\mu \nu}=a^{2}\left(\eta_{\mu \nu}+\sqrt{\kappa} \psi_{\mu \nu}\right) \\
\hat{g}^{\mu \nu} & =g^{\mu \nu}(\eta)+\delta g^{\mu \nu}=a^{-2}\left(\eta^{\mu \nu}-\sqrt{\kappa} a^{4} \psi^{\mu \nu}\right)+\mathcal{O}\left(\psi^{2}\right) \\
\hat{\phi} & =\Phi(\eta)+\phi
\end{aligned}
$$

where $\delta g_{\mu \nu} \equiv h_{\mu \nu}$ denotes the graviton field, $\psi_{\mu \nu}$ is the pseudo-graviton field and $\delta g^{\mu \nu}=$ $-h^{\mu \nu}+h_{\alpha}^{\mu} h^{\alpha \nu}+\mathcal{O}\left(h^{3}\right)$. Notice that indices on the pseudo-graviton field $\psi_{\mu \nu}$ are raised and lowered with the full background metric $g_{\mu \nu}(\eta)=a^{2} \eta_{\mu \nu}$. The background scalar field $\Phi$ is

homogeneous and thus only depends on (conformal) time. The background fields obey the 
tree level Friedmann equations and the scalar field equation of motion:

$$
\begin{aligned}
H^{2}-\frac{1}{D-1} \Lambda-\frac{\kappa}{(D-1)(D-2)}\left(\frac{1}{2 a^{2}} \Phi^{\prime 2}+V(\Phi)\right) & =0 \\
a^{-1} H^{\prime}+\frac{D-1}{2} H^{2}-\frac{1}{2} \Lambda+\frac{\kappa}{2(D-2)}\left(\frac{1}{2 a^{2}} \Phi^{\prime 2}-V(\Phi)\right) & =0 \\
\Phi^{\prime \prime}+(D-2) a H \Phi^{\prime}+a^{2} \frac{\partial V}{\partial \phi}(\Phi) & =0,
\end{aligned}
$$

from which one can derive the following identities

$$
\begin{aligned}
\sqrt{\kappa} \Phi^{\prime}=\sqrt{2(D-2) \epsilon} a H ; & \sqrt{\kappa} \Phi^{\prime \prime}=\sqrt{2(D-2) \epsilon}(1-\epsilon) a^{2} H^{2}+\mathcal{O}\left(\epsilon^{\prime}\right) \\
\sqrt{\kappa} \frac{\partial V}{\partial \phi}(\Phi)=-\sqrt{2(D-2) \epsilon}(D-1-\epsilon) H^{2} ; & \frac{\partial^{2} V}{\partial \phi^{2}}(\Phi)=2(D-1-\epsilon) \epsilon H^{2}+\mathcal{O}\left(\epsilon^{\prime}\right) .
\end{aligned}
$$

Notice that in slow roll inflation $\epsilon^{\prime}$ is non zero. In order to facilitate a comparison, we give here the slow roll expressions for the second and fourth relation (the first and third do not change)

$$
\begin{aligned}
\sqrt{\kappa} \Phi^{\prime \prime} & =\sqrt{\frac{2 \epsilon_{V}}{D-2}} a^{2} H^{2}\left(D+(D-4) \epsilon_{V}-\frac{(D-1)(D-2)}{2} \eta_{V}\right) \\
\frac{\partial^{2} V}{\partial \phi^{2}}(\Phi) & =\frac{(D-1)(D-2)}{2} \eta_{V} H^{2},
\end{aligned}
$$

in terms of the slow roll parameters

$$
\epsilon_{V} \equiv \frac{1}{\kappa}\left(\frac{\partial V}{V \partial \phi}\right)^{2} \quad ; \quad \eta_{V} \equiv \frac{2}{\kappa} \frac{\partial^{2} V}{V \partial \phi^{2}}
$$

Notice that in the slow roll approximation $\epsilon$ and $\epsilon_{V}$ are identical.

For the purpose of this paper we are only interested in the quadratic perturbations in the fields. After many partial integrations we find 1

$$
\begin{aligned}
\mathcal{L}^{(2)}= & a^{D+4} \psi^{\mu \nu}\left(\left(\square_{s}-\mathcal{W}\right)\left(\frac{1}{4} \delta_{\mu}^{\rho} \delta_{\nu}^{\sigma}-\frac{1}{8} \eta_{\mu \nu} \eta^{\rho \sigma}\right)+\mathcal{X} \delta_{\mu}^{0} \delta_{\nu}^{\rho} \delta_{0}^{\sigma}-\mathcal{Y} \eta_{\mu 0} \delta_{\nu}^{0} \eta^{\rho \sigma}\right) \psi_{\rho \sigma} \\
& -a^{D-2} \psi_{00}\left(\sqrt{\kappa} \Phi^{\prime \prime}\right) \phi+a^{D-2} \sqrt{\kappa} \eta^{\mu \nu} \psi_{\mu \nu} \mathcal{Z} \phi \\
& +\frac{1}{2} a^{D} \phi\left(\square_{s}-\frac{\partial^{2} V}{\partial \phi^{2}}(\Phi)+a^{-2} \Phi^{\prime 2} \kappa\right) \phi+\frac{1}{2} \sqrt{-g} g^{\alpha \beta} F_{\alpha} F_{\beta},
\end{aligned}
$$

\footnotetext{
${ }^{1}$ An analogous result can be found in Ref. [26. The main difference is that our result (24) includes also terms that vanish on-shell.
} 
where we defined

$$
\begin{aligned}
F_{\alpha} & =a^{2} \nabla_{\mu}\left(\psi_{\alpha}^{\mu}-\frac{1}{2} \delta_{\alpha}^{\mu} g^{\rho \sigma} \psi_{\rho \sigma}\right)-\phi \Phi^{\prime} \sqrt{\kappa} \delta_{\alpha}^{0} \\
\mathcal{W} & =2(D-2)\left[a^{-1} H^{\prime}+\frac{D-1}{2} H^{2}-\frac{1}{2} \Lambda+\frac{\kappa}{2(D-2)}\left(\frac{1}{2 a^{2}} \Phi^{\prime 2}-V(\Phi)\right)\right] \\
\mathcal{X} & =\left(\frac{1}{2} \kappa a^{-2} \Phi^{\prime 2}-\frac{D-2}{2}\left(H^{2}-a^{-1} H^{\prime}\right)\right) \\
\mathcal{Y} & =\left(\frac{1}{4} \kappa a^{-2} \Phi^{\prime 2}+\frac{D-2}{2} a^{-1} H^{\prime}\right) \\
\mathcal{Z} & =-\frac{1}{2}\left(\Phi^{\prime \prime}+(D-2) a H \Phi^{\prime}+\frac{\partial V}{\partial \phi}(\Phi) a^{2}\right)
\end{aligned}
$$

and

$$
\square_{s}=\frac{1}{\sqrt{-g}} \partial_{\mu} \sqrt{-g} g^{\mu \nu} \partial_{\nu}
$$

is the d'Alembertian as it acts on a scalar field. We add a gauge fixing term

$$
\mathcal{L}_{G F}=-\frac{1}{2} \sqrt{-g} g^{\alpha \beta} F_{\alpha} F_{\beta}
$$

and therefore we also need to add a ghost lagrangian

$$
\mathcal{L}_{\text {ghost }}=-\sqrt{-g} a^{-2} \bar{V}^{\mu} \delta F_{\mu},
$$

where we consider the change of $F_{\mu}$ under infinitesimal coordinate transformations $x^{\prime \mu}=$ $x^{\mu}+\sqrt{\kappa} V^{\mu}$. From $\phi\left(x^{\mu}\right)=\phi\left(x^{\prime \mu}\right)+\delta \phi$ and $\psi_{\mu \nu}\left(x^{\mu}\right)=\psi_{\mu \nu}\left(x^{\prime \mu}\right)+\delta \psi_{\mu \nu}$, we find up to first order in $V^{\mu}$ :

$$
\begin{aligned}
\delta \phi & =-\sqrt{\kappa} V^{0} \Phi^{\prime} \\
\delta \psi_{\mu \nu} & =-a^{-2}\left(g_{\alpha \nu} \partial_{\mu} V^{\alpha}+g_{\alpha \mu} \partial_{\nu} V^{\alpha}+2\left(\frac{a^{\prime}}{a}\right) g_{\mu \nu} V^{0}\right)
\end{aligned}
$$

and thus

$$
\mathcal{L}_{\text {ghost }}=a^{D} \eta_{\alpha \beta} \bar{V}^{\alpha}\left(\delta_{\mu}^{\beta} \square_{s}-(D-2)\left(H^{2}-a^{-1} H^{\prime}\right) \delta_{0}^{\beta} \delta_{\mu}^{0}+a^{-2} \kappa \Phi^{\prime 2} \delta_{0}^{\beta} \delta_{\mu}^{0}\right) V^{\mu},
$$

where $V$ and $\bar{V}$ are the ghost and anti-ghost fields. 
The quadratic lagrangian (24 25) contains mixing between the different components. The following field redefinition removes the mixing between $\psi_{i j}$ and $\psi_{00}$ and $\phi$ on-shell 2 .

$$
\begin{aligned}
& \psi_{i j}=z_{i j}+\frac{\delta_{i j}}{D-3} z_{00} \\
& \psi_{00}=z_{00} \\
& \psi_{0 i}=z_{0 i} .
\end{aligned}
$$

The resulting quadratic lagrangian (which is still valid off shell) can be written as

$$
\mathcal{L}^{(2)}+\mathcal{L}_{G F}+\mathcal{L}_{\text {ghost }}=\frac{1}{2} X_{i j}^{T} G^{i j k l} X_{k l}+\frac{1}{2} z_{0 i} \mathcal{D}_{\text {vector }}^{i j} z_{o j}+\bar{V}^{\alpha} \mathcal{D}_{\alpha \beta}^{\text {ghost }} V^{\beta}
$$

where

$$
\begin{aligned}
& X_{i j}=\left(\begin{array}{c}
z_{i j} \\
z_{00} \\
\phi
\end{array}\right) \\
& G^{i j k l}=\left(\begin{array}{ccc}
\mathcal{D}_{\text {tensor }}^{i j k l} & a^{D} \mathcal{Y} \delta^{i j} & a^{D-2} \sqrt{\kappa} \mathcal{Z} \delta^{i j} \\
a^{D} \mathcal{Y} \delta^{k l} & \mathcal{D}_{\text {scalar }} & a^{D-2} \sqrt{\kappa}\left(\frac{2}{D-3} \mathcal{Z}-\Phi^{\prime \prime}\right) \\
a^{D-2} \sqrt{\kappa} \mathcal{Z} \delta^{k l} & a^{D-2} \sqrt{\kappa}\left(\frac{2}{D-3} \mathcal{Z}-\Phi^{\prime \prime}\right) & \mathcal{D}_{\phi}
\end{array}\right) \\
& \mathcal{D}_{\text {tensor }}^{i j k l}=a^{D}\left(\square_{s}-\mathcal{W}\right)\left(\frac{1}{2} \delta^{i k} \delta^{j l}-\frac{1}{4} \delta^{i j} \delta^{k l}\right) \\
& \mathcal{D}_{\text {vector }}^{i j}=-a^{D}\left(\left(\square_{s}-\mathcal{W}\right)+2 \mathcal{X}\right) \eta^{i j} \\
& \mathcal{D}_{\text {scalar }}=a^{D}\left(\frac{D-2}{2(D-3)}\left(\square_{s}-\mathcal{W}\right)+2 \mathcal{X}+\frac{4}{D-3} \mathcal{Y}\right) \\
& \mathcal{D}_{\phi}=a^{D}\left(\square_{s}-\frac{\partial^{2} V}{\partial \phi^{2}}(\Phi)+a^{-2} \Phi^{\prime 2} \kappa\right) \\
& \mathcal{D}_{\alpha \beta}^{\text {ghost }}=a^{D}\left(\eta_{\alpha \beta} \square_{s}+2 \mathcal{X} \delta_{\alpha}^{0} \eta_{\beta 0}\right) .
\end{aligned}
$$

Note that $G^{i j k l}$ contains the tensor as well as the two scalar (gravitational and matter) kinetic operators.

\footnotetext{
${ }^{2}$ In the special case when $D=4$, this and Eq. (40) below agree with Ref. [26.
} 


\subsection{The propagators}

We shall now construct the propagators, associated to the various modes in (32). In general this will not be possible, due to the nontrivial dependence of the propagators on the background fields and the mixing between the different modes. Therefore we shall restrict ourselves in calculating the on-shell propagators. As will be clear from the discussion in section 4.2 this will be sufficient to calculate the one loop effective action. It will turn out that kinetic operators can be written in terms of

$$
\mathcal{D}_{n} \equiv \sqrt{-g}\left[\square_{s}-n\left(D-n-1+\frac{n(n-1)}{2} \epsilon\right)(1-\epsilon) H^{2}\right] \quad(n=0,1,2),
$$

with an associated propagator

$$
\mathcal{D}_{n} i \Delta_{n}(x ; \tilde{x})=i \delta^{D}(x-\tilde{x}) \quad(n=0,1,2) .
$$

The operator (34) is however nothing but the kinetic operator for the massless scalar field, with conformal coupling

$$
\xi=\frac{n\left(D-n-1+\frac{n(n-1)}{2} \epsilon\right)}{(D-1)(D-2 \epsilon)}(1-\epsilon)
$$

such that we can use the propagators calculated in the previous sections with the parameter $\nu$, given in (13) replaced by

$$
\nu_{n}^{2}=\left(\frac{D-1-\epsilon}{2(1-\epsilon)}\right)^{2}-\frac{n\left(D-n-1+\frac{n(n-1)}{2} \epsilon\right)}{1-\epsilon} .
$$

We find for the kinetic operators for the vector and the ghost on-shell

$$
\begin{aligned}
& \left.\mathcal{D}_{\text {vector }}^{i j}\right|_{\text {on shell }}=-\mathcal{D}_{1} \delta^{i j} \\
& \left.\mathcal{D}_{\mu \nu}^{\text {ghost }}\right|_{\text {on shell }}=\left(\bar{\eta}_{\mu \nu} \mathcal{D}_{0}+\delta_{\mu}^{0} \eta_{\nu_{0}} \mathcal{D}_{1}\right)
\end{aligned}
$$

and their associated propagators:

$$
\begin{aligned}
& i_{j} \Delta_{\text {vector }}^{k}=-\delta_{j}^{k} i \Delta_{1} \\
& i_{\alpha} \Delta_{\text {ghost }}^{\rho}=\left(\bar{\delta}_{\alpha}^{\rho} i \Delta_{0}+\delta_{\alpha}^{0} \delta_{0}^{\rho} i \Delta_{1}\right) .
\end{aligned}
$$


There is still mixing between $z_{00}$ and $\phi$. The on-shell part of the mixing can be removed by the following rotation

$$
\begin{aligned}
& X=R Y \\
& Y_{i j}=\left(\begin{array}{c}
z_{i j} \\
\chi \\
\nu
\end{array}\right) \\
& R=\left(\begin{array}{ccc}
1 & 0 & 0 \\
0 & \sqrt{\lambda} \cos (\theta) & -\sqrt{\lambda} \sin (\theta) \\
0 & \frac{1}{\sqrt{\lambda}} \sin (\theta) & \frac{1}{\sqrt{\lambda}} \cos (\theta)
\end{array}\right) \\
& \lambda=\sqrt{\frac{2(D-3)}{D-2}} \\
& \tan (2 \theta)=\frac{2 \sqrt{(D-3) \epsilon}}{D-3-\epsilon}, \quad \theta=\arccos \left(-\sqrt{\frac{\epsilon}{D-3+\epsilon}}\right) \text {. }
\end{aligned}
$$

This rotation reduces the on-shell part of the term $\frac{1}{2} X^{T} G X$ to

$$
\left.\frac{1}{2} Y_{i j}^{T} G_{\text {diag }}^{i j k l} Y_{k l}\right|_{\text {on shell }}=\frac{1}{2} Y_{i j}^{T}\left(\begin{array}{ccc}
\left(\frac{1}{2} \delta^{k i} \delta^{l j}-\frac{1}{4} \delta^{i j} \delta^{k l}\right) \mathcal{D}_{0} & 0 & 0 \\
0 & \frac{1}{\lambda} \mathcal{D}_{0} & 0 \\
0 & 0 & \frac{1}{\lambda} \mathcal{D}_{2}
\end{array}\right) Y_{k l}
$$

Thus the associated propagator matrix $\mathcal{M}_{\text {diag }}$, defined by

$$
\left.G_{\text {diag }} \mathcal{M}_{\text {diag }}(x ; \tilde{x})\right|_{\text {on shell }}=\mathbf{1} \delta^{D}(x-\tilde{x})
$$

is given by

$$
\begin{aligned}
& \left.\mathcal{M}_{\text {diag }}\right|_{\text {on shell }}=\left(\begin{array}{ccc}
{ }_{r s} \Delta_{k l} & 0 & 0 \\
0 & \lambda \Delta_{0} & 0 \\
0 & 0 & \lambda \Delta_{2}
\end{array}\right) \\
& i_{r s} \Delta_{k l}=\left(2 \delta_{r(k} \delta_{l) s}-\frac{2}{D-3} \delta_{r s} \delta_{k l}\right) i \Delta_{0} .
\end{aligned}
$$

To avoid confusion with the subscript diag, we have omitted the explicit Lorentz indices in $\mathcal{M}_{\text {diag }}$ and $G_{\text {diag. }}$. It now follows that the non-diagonal propagator matrix ${ }_{r s} \mathcal{M}_{k l}$ that inverts $G^{i j k l}$ on-shell is

$$
\begin{aligned}
\left.{ }_{r s} \mathcal{M}_{k l}\right|_{\text {on shell }} & =\left.R \mathcal{M}_{\text {diag }} R^{T}\right|_{\text {on shell }} \\
& =\left(\begin{array}{ccc}
{ }_{s} \Delta_{k l} & 0 & 0 \\
0 & \lambda^{2}\left(\cos ^{2}(\theta) \Delta_{0}+\sin ^{2}(\theta) \Delta_{2}\right) & \lambda \cos (\theta) \sin (\theta)\left(\Delta_{0}-\Delta_{2}\right) \\
0 & \lambda \cos (\theta) \sin (\theta)\left(\Delta_{0}-\Delta_{2}\right) & \left(\sin ^{2}(\theta) \Delta_{0}+\cos ^{2}(\theta) \Delta_{2}\right)
\end{array}\right) .
\end{aligned}
$$


This finishes the construction of all the propagators and we indeed find that all modes can be described in terms of $\Delta_{n}$.

\subsection{One-loop effective action}

In this section we shall first sketch using a simple example how to calculate the correction to the Friedmann equation due to the one-loop effective action. Afterwards we shall apply it to the case at hand. We consider as an example a model with an action

$$
S=S_{0}+S_{\chi}
$$

where $\chi$ is a quantum scalar field with an action $S_{\chi}=S_{\chi}[\chi]$ and $S_{0}$ is the classical action of any background fields (including for example the Einstein-Hilbert action). For any action $S_{\chi}$ that is quadratic in $\chi$,

$$
S_{\chi}=\int d^{D} x \frac{1}{2} \chi \mathcal{D}_{\chi} \chi
$$

one gets the following effective action

$$
\Gamma=S_{0}-i \ln \left(\frac{1}{\sqrt{\operatorname{Det}\left(\mathcal{D}_{\chi}\right)}}\right)=S_{0}+\frac{i}{2} \operatorname{Tr} \ln \left(\mathcal{D}_{\chi}\right) .
$$

Here the trace involves tracing over the Lorentz indices and space-time integration of the operator at coincidence [46] and $\mathcal{D}_{\chi}$ is the kinetic operator of the field.

While in principle one could - at least formally - evaluate the effective action, the object one is eventually interested in is the effective Friedmann equation, i.e. the equations of motion associated with the background metric. Moreover in the present case we need to work under the constraint that $\epsilon$ is constant. As long as $\dot{\epsilon}$ remains small, there is no problem with imposing such a constraint on the equations of motion. On the other hand, imposing this constraint on the level of the action typically changes the dynamics substantially. Therefore we shall not attempt to explicitly construct the effective action, but instead we shall directly calculate the effective Friedmann equation. By taking the functional derivative of the action with respect to the scale factor $a=a(\eta)$, we obtain the Einstein trace equation, that is the $-(00)+(D-1)(i i)$ component of the Einstein equation. The second Friedmann equation can then always be obtained by imposing the Bianchi identity. Thus we are interested in 
calculating

$$
\begin{aligned}
& \frac{\delta \Gamma}{\delta a}= \frac{\delta S_{0}}{\delta a}+\frac{i}{2} \frac{\delta}{\delta a} \operatorname{Tr} \ln \left(\mathcal{D}_{\chi}\right) \\
&=V a^{D-1}\left[\frac{D(D-1)(D-2)}{\kappa}\left(H^{2}-\frac{1}{D-1} \Lambda+\frac{2}{D} a^{-1} H^{\prime}\right)+(D-1) p_{M}-\rho_{M}\right] \\
& \quad+\frac{i}{2} \frac{\delta}{\delta a} \operatorname{Tr} \ln \left(\mathcal{D}_{\chi}\right),
\end{aligned}
$$

where $V=\int d^{D-1} x$ denotes the volume of space and we assumed that $S_{0}$ contains the Einstein-Hilbert action, and matter fields with an associated total pressure and energy $p_{M}$ and $\rho_{M}$. Notice that the quantum contribution $\frac{i}{2} \frac{\delta}{\delta a} \operatorname{Tr} \ln \left(\mathcal{D}_{\chi}\right)$ is by definition nothing but $V a^{D-1} g^{\mu \nu}\left\langle T_{\mu \nu}\right\rangle$, where $\left\langle T_{\mu \nu}\right\rangle$ is the one loop expectation value of the stress-energy tensor [46]. We now consider the calculation of this contribution. To be explicit, we shall assume that $\chi$ is a massless minimally coupled scalar and therefore

$$
\mathcal{D}_{\chi}=\sqrt{-g} \square
$$

with an associated propagator $i \Delta(x ; \tilde{x})$ which obeys

$$
\mathcal{D}_{\chi} i \Delta(x ; \tilde{x})=i \delta^{D}(x-\tilde{x}) .
$$

Instead of considering the $\chi$ field, it is convenient to use a rescaled field

$$
\hat{\chi}=a^{\frac{D}{2}-1} \chi
$$

with an associated kinetic operator and propagator

$$
\begin{aligned}
\hat{\mathcal{D}}_{\chi} & =\partial^{2}+\frac{(D-2)(D-4)}{4} \frac{a^{2}}{a^{2}}+\frac{D-2}{2} \frac{a^{\prime \prime}}{a} \\
i \hat{\Delta}(x ; \tilde{x}) & =a^{\frac{D}{2}-1} \hat{a}^{\frac{D}{2}-1} i \Delta(x ; \tilde{x}) .
\end{aligned}
$$

We shall see explicitly later that - up to a $D$ dimensional divergent delta function that does not contribute in dimensional regularization - we have that

$$
\frac{\delta}{\delta a} \operatorname{Tr} \ln \left(\mathcal{D}_{\chi}\right)=\frac{\delta}{\delta a} \operatorname{Tr} \ln \left(\hat{\mathcal{D}}_{\chi}\right) .
$$

We shall now show how to calculate the trace logarithm contribution to (48) due to the field $\hat{\chi}$. To be precise we give the exact coordinate dependence of each term indicated by $x^{\mu}, y^{\mu}$, 
and $z^{\mu}$

$$
\begin{aligned}
\frac{i}{2} \frac{\delta}{\delta a\left(z^{0}\right)} \operatorname{Tr} \ln \left(\hat{\mathcal{D}}_{\chi}(x)\right)= & \frac{i}{2} \operatorname{Tr}\left(\hat{\Delta}(x ; y)\left(\frac{\delta}{\delta a\left(z^{0}\right)} \hat{\mathcal{D}}_{\chi}(x)\right)\right) \\
= & \frac{i}{2} \int d^{D} x \int d^{D} y \hat{\Delta}(x ; y) \delta^{D}(x-y) \\
& {\left[\left(-\frac{(D-2)(D-4)}{2} \frac{a^{\prime}\left(x^{0}\right)^{2}}{a\left(x^{0}\right)^{3}}-\frac{D-2}{2} \frac{a^{\prime \prime}\left(x^{0}\right)}{a\left(x^{0}\right)^{2}}\right) \delta\left(x^{0}-z^{0}\right)\right.} \\
& \left.+\frac{(D-2)(D-4)}{2} \frac{a^{\prime}\left(x^{0}\right)}{a\left(x^{0}\right)^{2}} \partial_{x^{0}} \delta\left(x^{0}-z^{0}\right)+\frac{D-2}{2 a\left(x^{0}\right)} \partial_{x^{0}}^{2} \delta\left(x^{0}-z^{0}\right)\right] \\
= & \frac{1}{2} V\left[\left(-\frac{(D-2)(D-4)}{2} \frac{a^{\prime}\left(z^{0}\right)^{2}}{a\left(z^{0}\right)^{3}}-\frac{D-2}{2} \frac{a^{\prime \prime}\left(z^{0}\right)}{a\left(z^{0}\right)^{2}}\right) i \hat{\Delta}\left(z^{0} ; z^{0}\right)\right. \\
- & \left.\frac{(D-2)(D-4)}{2} \partial_{z^{0}}\left(\frac{a^{\prime}\left(z^{0}\right)}{a\left(z^{0}\right)^{2}} i \hat{\Delta}\left(z^{0} ; z^{0}\right)\right)+\frac{D-2}{2} \partial_{z^{0}}^{2}\left(\frac{1}{a\left(z^{0}\right)} i \hat{\Delta}\left(z^{0} ; z^{0}\right)\right)\right] \\
= & -\frac{D-2}{4} V a^{D-1} \square_{z} i \Delta(z ; z),
\end{aligned}
$$

where in going from the second to the third step we used the delta functions to change $\partial_{x^{0}}$ to $-\partial_{z^{0}}$ and we used that the propagators at coincidence are a function of time only. In the last step we used (52) to rewrite $i \hat{\Delta}$ in terms of $i \Delta$.

Since the trace of the one loop expectation value of the stress energy tensor for a scalar field is given by [46]

$$
\left\langle 0\left|T^{\mu}{ }_{\mu}\right| 0\right\rangle=\left(\frac{2-D}{4}+(D-1) \xi\right) \square i \Delta(x ; x),
$$

we see that indeed we have that

$$
\frac{i}{2} \frac{\delta}{\delta a} \operatorname{Tr} \ln \left(\hat{\mathcal{D}}_{\chi}\right)=V a^{D-1} g^{\mu \nu}\left\langle T_{\mu \nu}\right\rangle .
$$

Comparing this with Eq. (48) we see that this is exactly what one expects, justifying thus our procedure. The above example shows that indeed one can use the functional derivatives with respect to $a$ to evaluate the quantity that interests us. It also shows that the rescaling (51) does not influence the final result in the context of dimensional regularization. Notice that (48) is just an equation of motion. Therefore, after the variation is performed, in 
the second line of (54), we can safely evaluate all the quantities appearing on-shell. Hence we only need the propagators $i \Delta(y ; z)$ on-shell. This justifies our on-shell diagonalization procedure, based on which we constructed the propagators.

Now we apply this technique to the case at hand. The only difference is that there are more quantum fields. This however does not change the procedure. Up to an irrelevant constant, the effective action can be obtained from

$$
\exp [i \Gamma]=\int\left(\mathcal{D} h_{\mu \nu}\right)(\mathcal{D} \phi)\left(\mathcal{D} U^{\alpha}\right)\left(\mathcal{D} \bar{U}^{\alpha}\right) \exp \left(i\left(S^{(0)}+S^{(2)}\right)\right)
$$

where $U$ and $\bar{U}$ denote the unrescaled ghost fields associated with the graviton field $h_{\mu \nu}$. When written in terms of the rescaled fields this can be recast as 3

$$
\begin{aligned}
\exp [i \Gamma] & =\int\left(\mathcal{D} z_{i j}\right)\left(\mathcal{D} z_{0 i}\right)\left(\mathcal{D} z_{00}\right)(\mathcal{D} \phi)\left(\mathcal{D} V^{\alpha}\right)\left(\mathcal{D} \bar{V}^{\alpha}\right) \exp \left(i\left(S^{(0)}+S^{(2)}\right)\right) \\
& =\exp \left(i S^{(0)}\right) \frac{\mathcal{D}_{\alpha \beta}^{\text {ghost }}}{\sqrt{\operatorname{det}\left(\mathcal{D}_{\text {vector }}^{i j}\right) \operatorname{det}\left(G^{i j k l}\right)}},
\end{aligned}
$$

where

$$
\begin{aligned}
S^{(0)} & =\int d^{D} x \sqrt{-g}\left(\frac{1}{\kappa}(R-(D-2) \Lambda)-\frac{1}{2}(\partial \Phi)^{2}-V(\Phi)\right) \\
S^{(2)} & =\int d^{D} x \mathcal{L}^{(2)}
\end{aligned}
$$

and $\mathcal{L}^{(2)}$ is given in (32) and $(\partial \Phi)^{2}=-\Phi^{\prime 2}$. From Eq. (58) we obtain

$$
\begin{aligned}
\Gamma & =S^{(0)}+\frac{i}{2} \operatorname{Tr} \ln \left[\mathcal{D}_{\text {vector }}^{i j}\right]+\frac{i}{2} \operatorname{Tr} \ln \left[G^{i j k l}\right]-i \operatorname{Tr} \ln \left[\mathcal{D}_{\alpha \beta}^{\text {ghost }}\right] \\
& \equiv S^{(0)}+\Gamma_{1 L}
\end{aligned}
$$

from which we find the expression equivalent to (48) to be

$$
\begin{aligned}
\frac{\delta \Gamma}{\delta a} & =\frac{\delta S_{0}}{\delta a}+\frac{\delta \Gamma_{1 L}}{\delta a} \\
& =V a^{D-1}\left[\frac{D(D-1)(D-2)}{\kappa}\left(H^{2}-\frac{1}{D-1} \Lambda+\frac{2}{D} a^{-1} H^{\prime}\right)+(D-1) p_{M}-\rho_{M}\right]+\frac{\delta \Gamma_{1 L}}{\delta a}
\end{aligned}
$$

\footnotetext{
${ }^{3}$ Our field redefinition (31) has a Jacobian equal to one. Furthermore, the rescaling by $a^{2}$ of $\psi$ with respect to the 'true' graviton will contribute to the effective action as a $D$-dimensional delta function $\delta^{D}(0)$. Such a term does not contribute in dimensional regularization.
} 
where $p_{M}$ and $\rho_{M}$ are the pressure and energy density associated to the background scalar field matter, given by $\rho_{M}=\frac{1}{2} \dot{\Phi}^{2}+V(\Phi)$ and $p_{M}=\frac{1}{2} \dot{\Phi}^{2}-V(\Phi)$.

The one loop contribution can be written analogously to (54) as

$$
\frac{\delta \Gamma_{1 L}}{\delta a}=\int d^{D} x\left(\frac{i}{2}\left[{ }_{i} \Delta_{j}^{\text {vector }}\right](x ; x) \frac{\delta}{\delta a} \mathcal{D}_{\text {vector }}^{i j}+\frac{i}{2}\left[{ }_{i j} \mathcal{M}_{k l}\right] \frac{\delta}{\delta a} G^{i j k l}-i\left[{ }^{\alpha} \Delta_{\text {ghost }}^{\beta}\right](x ; x) \frac{\delta}{\delta a} \mathcal{D}_{\alpha \beta}^{\text {ghost }}\right) .
$$

The functional derivatives should naturally be taken on the off-shell kinetic operators. However, as soon as these derivatives are taken, we are simply left with an equation of motion. It is therefore completely valid to impose the background equations of motion at that point. Therefore the propagators in (62) can be evaluated on-shell and thus we can use the propagators as they are calculated in the previous section.

Instead of using the kinetic operators as given in (33), we rescale all of our fields as

$$
z_{\mu \nu} \rightarrow a^{1-D / 2} \hat{z}_{\mu \nu} \quad ; \quad \phi \rightarrow a^{1-D / 2} \hat{\phi}
$$

which is identical to the rescaling in Eq. (51). This rescaling changes the effective action (58) by a $D$ dimensional coincident delta function that does not contribute in dimensional regularization. With the following identity

$$
\phi \sqrt{-g} \square_{s} \phi=\hat{\phi}\left[\eta^{\alpha \beta} \partial_{\alpha} \partial_{\beta}+\frac{D-2}{2}\left(\frac{D}{2}-\epsilon\right) a^{2} H^{2}\right] \hat{\phi}
$$

we can easily calculate the kinetic operators of the rescaled fields. We will indicate these rescaled kinetic operators with a hat. The associated propagators are also easily obtained:

$$
\Delta(x ; \tilde{x})=(a \tilde{a})^{1-D / 2} \hat{\Delta}(x ; \tilde{x}) .
$$

We now shall consider one functional derivative in detail. The others are calculated similarly. We follow the same procedure as in the example considered at the start of this section. Our calculation proceeds analogously as in Eq. (54). We once again insert the explicit arguments 
$\eta=x^{0}$ and $\tilde{\eta}$ for the two coordinates respectively

$$
\begin{aligned}
& \int d^{D} x_{i j} \hat{\Delta}_{k l}(x ; x) \frac{\delta}{\delta a(\eta)} \hat{\mathcal{D}}_{\text {tensor }}^{i j k l}(\tilde{\eta}) \\
& =\int d^{D} x_{i j} \hat{\Delta}_{k l}(x ; x) \frac{\delta}{\delta a(\eta)}\left(\eta^{\alpha \beta} \partial_{\alpha} \partial_{\beta}+\frac{D-2}{2}\left(\frac{D}{2}-\epsilon(\tilde{\eta})\right) a(\tilde{\eta})^{2} H(\tilde{\eta})^{2}-a(\tilde{\eta})^{2} \mathcal{W}\right) \\
& \times\left(\frac{1}{2} \delta^{i k} \delta^{j l}-\frac{1}{4} \delta^{i j} \delta^{k l}\right) \\
& =\int d^{D} x_{i j} \hat{\Delta}_{k l}(x ; x) \frac{\delta}{\delta a(\eta)}\left(\eta^{\alpha \beta} \partial_{\alpha} \partial_{\beta}-\frac{D-2}{4}\left((3 D-16)\left(\frac{a(\tilde{\eta})^{\prime}}{a(\tilde{\eta})}\right)^{2}+6 \frac{a(\tilde{\eta})^{\prime \prime}}{a(\tilde{\eta})}\right)\right. \\
& \left.+(D-2) \Lambda a(\tilde{\eta})^{2}-\kappa\left(\frac{1}{2} \Phi^{\prime 2}-a(\tilde{\eta})^{2} V(\Phi)\right)\right)\left(\frac{1}{2} \delta^{i k} \delta^{j l}-\frac{1}{4} \delta^{i j} \delta^{k l}\right) \\
& =\int d^{D} x_{i j} \hat{\Delta}_{k l}(x ; x)\left(-\frac{D-2}{4}\left(2(3 D-16)\left(\frac{a^{\prime}(\tilde{\eta})}{a(\tilde{\eta})^{2}} \frac{\partial}{\partial \tilde{\eta}}-\frac{a^{\prime}(\tilde{\eta})^{2}}{a(\tilde{\eta})^{3}}\right)\right.\right. \\
& \left.+6\left(\frac{1}{a(\tilde{\eta})} \frac{\partial^{2}}{(\partial \tilde{\eta})^{2}}-\frac{a(\tilde{\eta})^{\prime \prime}}{a(\tilde{\eta})^{2}}\right)\right) \\
& +2(D-2) \Lambda a(\tilde{\eta})+2 \kappa V(\Phi) a(\tilde{\eta}))\left(\frac{1}{2} \delta^{i k} \delta^{j l}-\frac{1}{4} \delta^{i j} \delta^{k l}\right) \delta(\tilde{\eta}-\eta) \\
& =\int d^{D} x \delta(\tilde{\eta}-\eta)\left(-\frac{3(D-2)}{2} \frac{1}{a(\tilde{\eta})} \frac{\partial^{2}}{(\partial \tilde{\eta})^{2}}\right. \\
& +\frac{1}{2}(D-2)(3 D-10)\left(H \frac{\partial}{\partial \tilde{\eta}}+(1-\epsilon) H(\tilde{\eta})^{2} a(\tilde{\eta})\right) \\
& +2(D-2) \Lambda a(\tilde{\eta})+2 \kappa V(\Phi) a(\tilde{\eta}))\left(\frac{1}{2} \delta^{i k} \delta^{j l}-\frac{1}{4} \delta^{i j} \delta^{k l}\right)_{i j} \hat{\Delta}_{k l}(x ; x) \\
& =V\left(-\frac{3(D-2)}{2} \frac{1}{a} \partial_{\eta}^{2}+\frac{1}{2}(D-2)(3 D-10) H \partial_{\eta}\right. \\
& \left.+\frac{1}{2}(D-2)^{2}(7-3 \epsilon) H^{2} a\right)\left(\frac{1}{2} \delta^{i k} \delta^{j l}-\frac{1}{4} \delta^{i j} \delta^{k l}\right){ }_{i j} \hat{\Delta}_{k l}(x ; x) .
\end{aligned}
$$

In step three we integrated by parts and dropped the boundary terms because of the delta 
function. In step four we used the background equations of motion (20) for $\Phi$. This is justified, since corrections to those equations will be of order one loop and the above expression is already at order one loop. Therefore the error one is making is of two loop order.

The other functional derivatives we need are calculated similarly

$$
\begin{aligned}
& \frac{1}{V} \int{ }_{i} \hat{\Delta}_{j, \text { vector }}(x ; x) \frac{\delta}{\delta a} \hat{\mathcal{D}}_{\text {vector }}^{i j}=\left(\frac{D-2}{2} \frac{1}{a} \partial_{\eta}^{2}-\frac{1}{2}(D-2)(3 D-2) H \partial_{\eta}\right. \\
& \left.-\frac{1}{2}(D-2)((3 D+2)(1-\epsilon)+4(D-2)) a H^{2}\right) \delta^{i j}{ }_{i} \hat{\Delta}_{j, \text { vector }}(x ; x) \\
& \frac{1}{V} \int \hat{\mathcal{M}}_{(1,1)}(x ; x) \frac{\delta}{\delta a} \hat{\mathcal{D}}_{\text {scalar }}=\frac{D-2}{2(D-3)}\left(\frac{D+2}{2} \frac{1}{a} \partial_{\eta}^{2}+\frac{3}{2}\left(D^{2}-4\right) H \partial_{\eta}\right. \\
& \left.+\frac{1}{2}(D-2)((7 D+2)-(3 D+10) \epsilon) H^{2} a\right) \hat{\mathcal{M}}_{(1,1)}(x ; x) \\
& \frac{1}{V} \int \hat{\mathcal{M}}_{(2,2)}(x ; x) \frac{\delta}{\delta a} \hat{\mathcal{D}}_{\phi}=\left(\frac{D-2}{2} \frac{1}{a} \partial_{\eta}^{2}-\frac{1}{2}(D-2)^{2} H \partial_{\eta}-\left(\frac{1}{2}(D-2)^{2}(1-\epsilon)\right.\right. \\
& \left.+4(D-1-\epsilon) \epsilon) a H^{2}\right) \hat{\mathcal{M}}_{(2,2)}(x ; x) \\
& \frac{1}{V} \int \alpha \hat{\Delta}_{\text {ghost }}^{\beta} \frac{\delta}{\delta a} \hat{\mathcal{D}}_{\alpha \beta}^{\text {ghost }}=\left(\frac{D-2}{2} \frac{1}{a} \partial_{\eta}^{2}-\frac{1}{2}(D-2)^{2} H \partial_{\eta}-\frac{1}{2}(D-2)^{2}(1-\epsilon) H^{2} a\right) \eta_{\alpha \beta}{ }^{\alpha} \hat{\Delta}_{\text {ghost }}^{\beta} \\
& +\left((D-2) \frac{1}{a} \partial_{\eta}^{2}+4(D-2) H \partial_{\eta}+4(D-2)(1-\epsilon) H^{2} a\right) \delta_{\alpha}^{0} \eta_{\beta 0}{ }^{\alpha} \hat{\Delta}_{\text {ghost }}^{\beta} \\
& \frac{1}{V} \int \hat{\mathcal{M}}_{(1,2)}(x ; x) \frac{\delta}{\delta a} \sqrt{\kappa}\left(\frac{2}{D-3} \mathcal{Z}-\Phi^{\prime \prime}\right) \\
& =\frac{\sqrt{2(D-2) \epsilon}}{D-3}\left((D-2) H \partial_{\eta}+(3 D-4-D \epsilon) H^{2} a\right) \hat{\mathcal{M}}_{(1,2)}(x ; x) .
\end{aligned}
$$

Here we indicated with $\hat{\mathcal{M}}_{(n, m)}(x ; x)$ the $(n, m)$ component of the propagator matrix $\hat{\mathcal{M}}$ (44). The last thing we need before we can calculate the one loop contribution (62) are the propagators at coincidence and their derivatives. Since all propagators are related to the propagator $\hat{\Delta}_{n}$, we only need that one.

\subsubsection{Infinite volume contribution}

The propagators that we use are split in two parts (14): the infinite volume part and the corrections due to the infrared cut-off. 
We first consider the infinite volume contribution (15). Taking the $y \rightarrow 0$ limit of (15) and dropping the $D$ dependent powers of $y$ that do not contribute in dimensional regularization we obtain (we drop the subscript $\infty$ )

$$
\begin{aligned}
i \hat{\Delta}_{n}(x ; x)=a^{D-2} i \Delta_{n}(x ; x) & =|1-\epsilon|^{D-2}(a H)^{D-2} \frac{\Gamma\left(1-\frac{D}{2}\right)}{(4 \pi)^{\frac{D}{2}}} \frac{\Gamma\left(\frac{D-1}{2}+\nu_{D, n}\right) \Gamma\left(\frac{D-1}{2}-\nu_{D, n}\right)}{\Gamma\left(\frac{1}{2}+\nu_{D, n}\right) \Gamma\left(\frac{1}{2}-\nu_{D, n}\right)} \\
\frac{\partial}{\partial \eta} i \hat{\Delta}_{n}(x ; x) & =H a(D-2)(1-\epsilon) i \hat{\Delta}_{n}(x ; x) \\
\left(\frac{\partial}{\partial \eta}\right)^{2} i \hat{\Delta}_{n}(x ; x) & =H^{2} a^{2}(D-1)(D-2)(1-\epsilon)^{2} i \hat{\Delta}_{n}(x ; x) .
\end{aligned}
$$

We can now collect all the terms of (62). Using (39), (444), (66), (67) and (68) we obtain for the vector and the ghost

$$
\begin{aligned}
& \frac{1}{V} \int \frac{i}{2}{ }_{i} \hat{\Delta}_{j}^{\text {vector }}(x ; x) \frac{\delta}{\delta a} \hat{\mathcal{D}}_{\text {vector }}^{i j}= \frac{1}{4}(D-1)(D-2)[2(D-1)(D+2) \\
&\left.-\left(D^{2}+D+2\right) \epsilon-(D-1)(D-2) \epsilon^{2}\right] a H^{2} i \hat{\Delta}_{1}(x ; x) \\
& \frac{1}{V} \int-i^{\alpha} \hat{\Delta}_{\text {ghost }}^{\beta}(x ; x) \frac{\delta}{\delta a} \hat{\mathcal{D}}_{\alpha \beta}^{\text {ghost }}=H^{2} a \frac{1}{2}(D-1)^{2}(D-2)^{2} \epsilon(1-\epsilon) i \hat{\Delta}_{0}(x ; x) \\
&-\frac{1}{2}(D-1)(D-2)(1-\epsilon)(2(D+2)-3(D-2) \epsilon) a H^{2} i \hat{\Delta}_{1}(x ; x) .
\end{aligned}
$$

The last term evaluates to

$$
\begin{aligned}
\frac{1}{V} \int \frac{i}{2}{ }_{i} j \hat{\mathcal{M}}_{k l}(x ; x) \frac{\delta}{\delta a} \hat{G}^{i j k l} & =\frac{1}{V} \int \frac{i}{2}{ }_{i j} \tilde{\Delta}_{k l}(x ; x) \frac{\delta}{\delta a} \hat{\mathcal{D}}_{\text {tensor }}^{i j k l} \\
+ & \frac{1}{2} \lambda^{2}\left(\cos (\theta)^{2} i \hat{\Delta}_{0}+\sin (\theta)^{2} i \hat{\Delta}_{2}\right) \frac{\delta}{\delta a} \hat{\mathcal{D}}_{\text {scalar }} \\
+ & \left.\lambda \cos (\theta) \sin (\theta)\left(i \hat{\Delta}_{0}-i \hat{\Delta}_{2}\right) \frac{\delta}{\delta a}\left(\sqrt{\kappa} \frac{2}{D-3} \mathcal{Z}-\sqrt{\kappa} \Phi^{\prime \prime}\right)\right) \\
+ & \frac{1}{2}\left(\sin (\theta)^{2} i \hat{\Delta}_{0}+\cos (\theta)^{2} i \hat{\Delta}_{2}\right) \frac{\delta}{\delta a} \hat{\mathcal{D}}_{\phi} .
\end{aligned}
$$

The contribution from the tensor is

$$
\frac{1}{8} D(D-1)(D-2)^{2}[(1+3 D)-3(D-1) \epsilon] \epsilon a H^{2} i \hat{\Delta}_{0}(x ; x),
$$


while the terms in (70) multiplying $i \hat{\Delta}_{0}$ contribute as

$$
\begin{aligned}
\frac{\epsilon}{4(D-3+\epsilon)}[ & -(D-1)(D-3)\left(D^{2}-8 D+4\right)+\left[D(D-2)\left(D^{2}-11 D+14\right)+8\right] \epsilon \\
& \left.+(D-1)(D-2)(D+2) \epsilon^{2}\right] a H^{2} i \hat{\Delta}_{0}(x ; x)
\end{aligned}
$$

Finally the terms in (70) multiplying $i \hat{\Delta}_{2}$ contribute as

$$
\begin{aligned}
& \frac{1}{4(D-3+\epsilon)}\left[4(D-1)(D-2)(D-3)(D+3)-\left(5 D^{4}-20 D^{3}-9 D^{2}+68 D-36\right) \epsilon\right. \\
& \left.\quad+\left(D^{4}-5 D^{3}-4 D^{2}+24 D-32\right) \epsilon^{2}+\left(D^{3}-5 D^{2}+8 D+4\right) \epsilon^{3}\right] a H^{2} i \hat{\Delta}_{2}(x ; x) .
\end{aligned}
$$

Putting (69 (73) and (62) together and expanding the result around $D=4$ we obtain the following non renormalized one loop contributions to the Friedmann trace equation,

$$
\begin{aligned}
& \frac{1}{V a^{D-1} H^{4}} \frac{\delta \Gamma_{1 L}^{0}}{\delta a}=\frac{\epsilon\left(63 \epsilon^{2}+2 \epsilon-105\right)}{64 \pi^{2}(1+\epsilon)}(1-\epsilon)^{2}\left(4 \nu_{0}^{2}-1\right)\left(\frac{2}{D-4}+\gamma_{E}+\ln \left(\frac{(1-\epsilon)^{2} H^{2}}{4 \pi \mu^{2}}\right)\right. \\
& \left.+\psi\left(\frac{1}{2}+\nu_{0}\right)+\psi\left(\frac{1}{2}-\nu_{0}\right)+4 \frac{4 \nu_{0} \nu_{0}^{\prime}-1}{4 \nu_{0}^{2}-1}\right) \\
& +\frac{\epsilon}{32 \pi^{2}(1+\epsilon)^{2}}(1-\epsilon)^{2}\left(4 \nu_{0}^{2}-1\right)\left(93 \epsilon^{3}+90 \epsilon^{2}-169 \epsilon-122\right) \\
& \frac{1}{V a^{D-1} H^{4}} \frac{\delta \Gamma_{1 L}^{1}}{\delta a}=\frac{3\left(9 \epsilon^{2}-7 \epsilon-6\right)}{64 \pi^{2}}(1-\epsilon)^{2}\left(4 \nu_{1}^{2}-1\right)\left(\frac{2}{D-4}+\gamma_{E}+\ln \left(\frac{(1-\epsilon)^{2} H^{2}}{4 \pi \mu^{2}}\right)\right. \\
& \left.+\psi\left(\frac{1}{2}+\nu_{1}\right)+\psi\left(\frac{1}{2}-\nu_{1}\right)+4 \frac{4 \nu_{1} \nu_{1}^{\prime}-1}{4 \nu_{1}^{2}-1}\right) \\
& +\frac{1}{64 \pi^{2}}(1-\epsilon)^{2}\left(4 \nu_{1}^{2}-1\right)\left(51 \epsilon^{2}-17 \epsilon-54\right) \\
& \frac{1}{V a^{D-1} H^{4}} \frac{\delta \Gamma_{1 L}^{2}}{\delta a}=-\frac{(5 \epsilon-6)\left(\epsilon^{2}-2 \epsilon-7\right)}{64 \pi^{2}(1+\epsilon)}(1-\epsilon)^{2}\left(4 \nu_{2}^{2}-1\right)\left(\frac{2}{D-4}+\gamma_{E}+\ln \left(\frac{(1-\epsilon)^{2} H^{2}}{4 \pi \mu^{2}}\right)\right. \\
& \left.+\psi\left(\frac{1}{2}+\nu_{2}\right)+\psi\left(\frac{1}{2}-\nu_{2}\right)+4 \frac{4 \nu_{2} \nu_{2}^{\prime}-1}{4 \nu_{2}^{2}-1}\right) \\
& +\frac{1}{64 \pi^{2}(1+\epsilon)^{2}}(1-\epsilon)^{2}\left(4 \nu_{2}^{2}-1\right)\left(3 \epsilon^{4}+13 \epsilon^{3}-83 \epsilon^{2}+35 \epsilon+30\right) \text {. }
\end{aligned}
$$


Here $\nu_{n}$ indicates the index $\nu_{n}$ given in (37) with $D=4$ and $\nu_{n}^{\prime}$ is $\left.\frac{d}{d D} \nu_{D, n}\right|_{D=4} \cdot \gamma_{E}$ is the Euler constant and we used the following expansions of the propagators (68)

$$
\begin{aligned}
a H^{2} i \hat{\Delta}_{n}(x ; x) & =a^{D-1}|1-\epsilon|^{D-2} H^{D} \frac{\Gamma\left(1-\frac{D}{2}\right)}{(4 \pi)^{\frac{D}{2}}}\left(\left(\frac{D-3}{2}\right)^{2}-\nu_{n}^{2}\right) \\
& \times\left[1+\frac{D-4}{2}\left(\psi\left(\frac{1}{2}+\nu_{n}\right)+\psi\left(\frac{1}{2}-\nu_{n}\right)\right)\right] \\
|1-\epsilon|^{D-2} H^{D} \frac{\Gamma\left(1-\frac{D}{2}\right)}{(4 \pi)^{\frac{D}{2}}} & =\frac{(1-\epsilon)^{2} H^{4}}{16 \pi^{2}} \\
& \times\left(\frac{2 \mu^{D-4}}{D-4}+\gamma_{E}-1+\ln \left(\frac{H^{2}}{\mu^{2}}\right)+\ln \left(\frac{(1-\epsilon)^{2}}{4 \pi}\right)\right),
\end{aligned}
$$

plus terms that vanish in $D=4$. Here $\psi(z)=(d / d z) \ln (\Gamma(z))$ is the digamma function and $\mu$ is an arbitrary renormalization scale introduced for later convenience. If we use the explicit expression for $\nu_{n}$, we can add all terms together and obtain for the functional derivative of the effective action

$$
\begin{aligned}
\frac{1}{V a^{D-1} H^{4}} \frac{\delta \Gamma_{1 L}}{\delta a} & =\frac{1}{V a^{D-1} H^{4}} \frac{\delta \Gamma_{1 L}^{0}}{\delta a}+\frac{1}{V a^{D-1} H^{4}} \frac{\delta \Gamma_{1 L}^{1}}{\delta a}+\frac{1}{V a^{D-1} H^{4}} \frac{\delta \Gamma_{1 L}^{2}}{\delta a} \\
& =-\frac{\epsilon\left(186-149 \epsilon-11 \epsilon^{2}+10 \epsilon^{3}\right)}{8 \pi^{2}} \frac{\mu^{D-4}}{D-4} \\
& -\frac{\epsilon}{16 \pi^{2}}\left[\left(108+62 \epsilon-153 \epsilon^{2}+27 \epsilon^{3}\right)+\left(186-149 \epsilon-11 \epsilon^{2}+10 \epsilon^{3}\right)\right. \\
& +\frac{\epsilon\left(63 \epsilon^{2}+2 \epsilon-105\right)}{64 \pi^{2}(1+\epsilon)}(1-\epsilon)^{2}\left(4 \nu_{0}^{2}-1\right)\left(\psi\left(\frac{1}{2}+\nu_{0}\right)+\psi\left(\frac{1}{2}-\nu_{0}\right)\right) \\
& +\frac{3\left(9 \epsilon^{2}-7 \epsilon-6\right)}{64 \pi^{2}}(1-\epsilon)^{2}\left(4 \nu_{1}^{2}-1\right)\left(\psi\left(\frac{1}{2}+\nu_{1}\right)+\psi\left(\frac{1}{2}-\nu_{1}\right)\right) \\
& -\frac{(5 \epsilon-6)\left(\epsilon^{2}-2 \epsilon-7\right)}{64 \pi^{2}(1+\epsilon)}(1-\epsilon)^{2}\left(4 \nu_{2}^{2}-1\right)\left(\psi\left(\frac{1}{2}+\nu_{2}\right)+\psi\left(\frac{1}{2}-\nu_{2}\right)\right) .
\end{aligned}
$$

We kept the digamma functions in terms of $\nu$, since then it will be more clear how to add the correction terms to regulate the infrared. In (77), all infrared power-law divergences 
are (incorrectly!) subtracted by the automatic subtraction of dimensional regularization. The infrared logarithmic divergences are still there and they appear through the poles in the digamma functions. These issues will be corrected by adding the correction terms, but before adding the correction terms, we shall first renormalize the expression (77).

\subsubsection{Renormalization}

The contribution (77) contains a $1 /(D-4)$ divergence and therefore needs to be renormalized. If we take our approximation that $\epsilon=$ constant literally, the divergence is of the form constant $\times H^{4} a^{D-1}$. However in a more realistic treatment, $\epsilon$ is a dynamical parameter and our result is expected to be correct up to zeroth order in $\dot{\epsilon}$. Such an approach, using a space-time of locally constant $\epsilon$ is a generalization of the often considered locally de Sitter space-time [49, 50]. In this more realistic case, the $\epsilon$ structure of the divergent term should be taken into account and subtracted accordingly. Therefore if $\epsilon$ is varying slowly enough, such that (77) remains approximately valid, we still need a counter lagrangian that produces the same $\epsilon$ structure as the divergence in (77), in order for the theory to be renormalized at all times. For this purpose many terms can be used, and indeed many terms are reported in the literature [14, 9, 47]. However we cannot simply use these terms, since counterterms are dependent on the gauge fixing used [48] and as far as we know, no general calculations have been done using our gauge fixing (27). Calculations using (27) have been done however in the special case of de Sitter space [28, 44], considering both scalar and graviton loops. From these works and also e.g. from [13] it follows that the one loop contribution in this limit should be finite. Since the de Sitter limit is $\epsilon \rightarrow 0$, this agrees with (77). To ensure that the one loop contribution due to gravitons in the de Sitter case vanishes we need in that limit a counterterm

$$
\sqrt{-g}\left(H^{2}-\frac{\Lambda}{D-1}\right)^{2}
$$

which in our more general case becomes

$$
\mathcal{L}_{C T 1}=\sqrt{-g} a_{0}\left(R-\frac{D}{D-2}(\kappa V(\Phi)+(D-2) \Lambda)\right)^{2}
$$

where $a_{0}$ is a constant. This follows from the fact that the cosmological constant can always be seen as a part of the scalar potential and thus for an invariant counterterm they should come together. Moreover from [44] it follows that we also need a counterterm of the form $\sqrt{-\hat{g}} H^{2} \kappa \hat{g}^{\mu \nu}\left(\partial_{\mu} \hat{\phi}\right)\left(\partial_{\nu} \hat{\phi}\right)$ to vanish. From Eq. (9) it follows that for our case this generalizes to

$$
\mathcal{L}_{C T 2}=\sqrt{-g} a_{1}\left(R g^{\mu \nu}-D R^{\mu \nu}\right) \kappa\left(\partial_{\mu} \Phi\right)\left(\partial_{\nu} \Phi\right)
$$


with $a_{1}$ a constant. Finally, also from [44] it follows that a counterterm $\sqrt{-g} \kappa(\square \Phi)^{2}$ should not appear.

Therefore a reasonable choice for the counter-lagrangian is

$$
\begin{aligned}
\mathcal{L}_{c}=\sqrt{-g}[ & a_{0}\left(R-\frac{D}{D-2}(\kappa V+(D-2) \Lambda)\right)^{2}+a_{1}\left(R g^{\mu \nu}-D R^{\mu \nu}\right) \kappa \partial_{\mu} \Phi \partial_{\nu} \Phi \\
& \left.+a_{2} \frac{\partial^{2} V(\Phi)}{\partial \Phi^{2}} R+a_{3} \kappa g^{\mu \nu}\left(\partial_{\mu} \Phi\right)\left(\partial_{\nu} \Phi\right) \frac{\partial^{2} V(\Phi)}{\partial \Phi^{2}}\right] .
\end{aligned}
$$

We stress that the counter-lagrangian (81) for the purpose of this calculation could be chosen differently. There are many other terms with the correct dimensionality that could have been used [47]. Since the divergence (77) gives only four constraints (one for each power of $\epsilon$ ), we at present can fix only 4 coefficients. This does not mean that the counter-lagrangian is arbitrary. Different types of calculations could fix the counter-lagrangian uniquely, as it is for example done in Ref. [9]. However apart from the two cases mentioned above, we do not know of any calculation in our gauge, which we could use to further specify our counter-lagrangian. Thus the 'true' counter-lagrangian corresponding to the theory will probably contain different counterterms than (81). However, these different counterterms do not change the conclusions of this paper. The only effect would be that in Eq. (85) the origin of the $\beta_{i}$ 's changes, but not the fact that they are essentially arbitrary. The terms in 
our counter-lagrangian (81) contribute as follows to the Friedmann trace equation

$$
\begin{aligned}
& \frac{1}{V} \frac{\delta}{\delta a} \int d^{D} x \sqrt{-g}\left(R-\frac{D}{D-2}(\kappa V+(D-2) \Lambda)\right)^{2} \\
& =a^{D-1} H^{4}(D-2) \epsilon\left(8 \epsilon(2+3 \epsilon)+D^{2}(4+9 \epsilon)-2 D\left(2+13 \epsilon+12 \epsilon^{2}\right)\right)+\mathcal{O}\left(\epsilon^{\prime}\right) \\
& =a^{D-1} H^{4}\left(16 \epsilon\left(6+7 \epsilon-9 \epsilon^{2}\right)+4 \epsilon\left(26+37 \epsilon-30 \epsilon^{2}\right)(D-4)+\mathcal{O}\left((D-4)^{2}, \epsilon^{\prime}\right)\right) \\
& \frac{1}{V} \frac{\delta}{\delta a} \int d^{D} x \kappa \sqrt{-g}\left(R g^{\mu \nu}-D R^{\mu \nu}\right)\left(\partial_{\mu} \Phi\right)\left(\partial_{\nu} \Phi\right) \\
& =2 a^{D-1} H^{4}(D-1)(D-2)^{2} \epsilon\left((D-1)(D-6 \epsilon)+6 \epsilon^{2}\right)+\mathcal{O}\left(\epsilon^{\prime}\right) \\
& =a^{D-1} H^{4}\left(144 \epsilon(1-\epsilon)(2-\epsilon)+24 \epsilon\left(23-30 \epsilon+8 \epsilon^{2}\right)(D-4)+\mathcal{O}\left((D-4)^{2}, \epsilon^{\prime}\right)\right. \\
& \frac{1}{V} \frac{\delta}{\delta a} \int d^{D} x \sqrt{-g} \frac{\partial^{2} V(\Phi)}{\partial \Phi^{2}} R \\
& =a^{D-1} H^{4}(D-1)(D-1-\epsilon) 2 \epsilon\left(D(D-2)-2(3 D-4) \epsilon+12 \epsilon^{2}\right)+\mathcal{O}\left(\epsilon^{\prime}\right) \\
& =a^{D-1} H^{4}\left(24 \epsilon(3-\epsilon)\left(2-4 \epsilon+3 \epsilon^{2}\right)\right. \\
& \left.+4 \epsilon\left(51-88 \epsilon+53 \epsilon^{2}-6 \epsilon^{3}\right)(D-4)\right)+\mathcal{O}\left((D-4)^{2}, \epsilon^{\prime}\right) \\
& \frac{1}{V} \frac{\delta}{\delta a} \int d^{D} x \kappa \sqrt{-g} g^{\mu \nu}\left(\partial_{\mu} \Phi\right)\left(\partial_{\nu} \Phi\right) \frac{\partial^{2} V(\Phi)}{\partial \Phi^{2}} \\
& =-4 a^{D-1} H^{4}(D-2)^{2} \epsilon^{2}(D-1-\epsilon)+\mathcal{O}\left(\epsilon^{\prime}\right) \\
& =a^{D-1} H^{4}\left(-16 \epsilon^{2}(3-\epsilon)-16 \epsilon^{2}(4-\epsilon)(D-4)\right)+\mathcal{O}\left((D-4)^{2}, \epsilon^{\prime}\right),
\end{aligned}
$$

where we used (91) and once again we used the background equations of motion (20) and (21). We find that all divergencies cancel if

$$
\begin{array}{rlrl}
a_{0}=\frac{37}{960 \pi^{2}} \frac{\mu^{D-4}}{D-4}+a_{0}^{f} & ; & a_{1}=\frac{49}{640 \pi^{2}} \frac{\mu^{D-4}}{D-4}+a_{1}^{f} \\
a_{2}=-\frac{5}{288 \pi^{2}} \frac{\mu^{D-4}}{D-4}+a_{2}^{f} \quad ; & a_{3}=-\frac{43}{480 \pi^{2}} \frac{\mu^{D-4}}{D-4}+a_{3}^{f},
\end{array}
$$

where the $a_{i}^{f}(i=0,1,2,3)$ indicates a possible finite part. Adding the contribution from the counterterms (82) to the one loop contribution (77), we obtain the following renormalized 
contribution

$$
\begin{aligned}
\frac{1}{a^{3} V} \frac{\Gamma_{1 L, \text { ren }}}{\delta a} & =\frac{H^{4}}{16 \pi^{2}}\left[\beta_{1} \epsilon+\beta_{2} \epsilon^{2}+\beta_{3} \epsilon^{3}+\beta_{4} \epsilon^{4}\right. \\
& -\epsilon\left(186-149 \epsilon-11 \epsilon^{2}+10 \epsilon^{3}\right)\left(\ln \left(\frac{(1-\epsilon)^{2} H^{2}}{4 \pi \mu^{2}}\right)\right) \\
& +\frac{\epsilon\left(63 \epsilon^{2}+2 \epsilon-105\right)}{4(1+\epsilon)}(1-\epsilon)^{2}\left(4 \nu_{0}^{2}-1\right)\left(\psi\left(\frac{1}{2}+\nu_{0}\right)+\psi\left(\frac{1}{2}-\nu_{0}\right)\right) \\
& +\frac{3\left(9 \epsilon^{2}-7 \epsilon-6\right)}{4}(1-\epsilon)^{2}\left(4 \nu_{1}^{2}-1\right)\left(\psi\left(\frac{1}{2}+\nu_{1}\right)+\psi\left(\frac{1}{2}-\nu_{1}\right)\right) \\
& \left.-\frac{(5 \epsilon-6)\left(\epsilon^{2}-2 \epsilon-7\right)}{4(1+\epsilon)}(1-\epsilon)^{2}\left(4 \nu_{2}^{2}-1\right)\left(\psi\left(\frac{1}{2}+\nu_{2}\right)+\psi\left(\frac{1}{2}-\nu_{2}\right)\right)\right] .
\end{aligned}
$$

Here the parameters $\beta_{i}(i=1,2,3,4)$ are given in terms of the finite coefficients $\alpha_{i}^{f}$,

$$
\begin{aligned}
& \beta_{1}=16 \pi^{2} \times 12\left[8 a_{0}^{f}+24 a_{1}^{f}+12 a_{2}^{f}\right]-186 \gamma_{E}+\frac{1727}{3} \\
& \beta_{2}=16 \pi^{2} \times 4\left[28 a_{0}^{f}-108 a_{1}^{f}-84 a_{2}^{f}-12 a_{3}^{f}\right]+149 \gamma_{E}-\frac{5969}{9} \\
& \beta_{3}=16 \pi^{2} \times 4\left[-36 a_{0}^{f}+36 a_{1}^{f}+78 a_{2}^{f}+4 a_{3}^{f}\right]+11 \gamma_{E}+\frac{10457}{45} \\
& \beta_{4}=16 \pi^{2}\left[-72 a_{2}^{f}\right]-10 \gamma_{E}-\frac{61}{3} .
\end{aligned}
$$

All $\beta_{i}$ 's $(i=1,2,3,4)$ in Eq. (85) are free parameters that remain undetermined until they are fixed by experiment.

\subsubsection{Correction terms}

The effective action (85) is divergent for half integer values of the parameters $\nu_{i} \geq 3 / 2$ (notice that the pole at $\nu_{i}=1 / 2$ is cancelled due to the pre-factor)

$$
\nu_{i}=3 / 2+N \quad ; \quad N=\{0,1,2 \ldots\} .
$$

The reason is that the propagators used do not describe the infrared physics correctly and we need to add the correction terms $\delta i \Delta_{N}$ and $\delta i \Delta^{N}$ as given in (16). For concreteness we shall consider the late time behavior in an accelerating space-time, since in that case we expect the most significant backreaction. In an accelerating space-time, $\eta$ goes to zero at 
late times, and thus we do not care about the $\delta i \Delta^{N}$ corrections, since they quickly become insignificant in that case. Since the correction terms are ultraviolet finite, we can put $D=4$ in all terms. Following the same procedure as in section 4.2.1, we find for the coincident limit and its derivatives

$$
\begin{aligned}
\delta i \hat{\Delta}_{N, n}(x ; x) & =A_{N, n}(a H)^{2} z_{0}^{2 N+3-2 \nu_{n}} \\
\frac{\partial}{\partial \eta} \delta i \hat{\Delta}_{N, n}(x ; x) & =-\left(1+2 N-2 \nu_{n}\right)(1-\epsilon)(a H) \delta i \hat{\Delta}_{N, n}(x ; x) \\
\left(\frac{\partial}{\partial \eta}\right)^{2} \delta i \hat{\Delta}_{N, n}(x ; x) & =\left(1+2 N-2 \nu_{n}\right)\left(2 N-2 \nu_{n}\right)(1-\epsilon)(a H) \delta i \hat{\Delta}_{N, n}(x ; x),
\end{aligned}
$$

where $\nu_{n}$ is given in (37) and a subscript $n$ implies that the quantity is evaluated with $\nu=\nu_{n}$. We also defined

$$
A_{N, n} \equiv-\frac{1}{4 \pi^{5 / 2}} \frac{1}{3+2 N-2 \nu_{n}} \frac{\Gamma\left(\nu_{n}-N\right) \Gamma\left(2 \nu_{n}-N\right)}{\Gamma\left(\frac{1}{2}+\nu_{n}-N\right) \Gamma(N+1)}(1-\epsilon)^{2} .
$$

We find for the corrections due to the vector and the ghost (67)

$$
\begin{aligned}
\frac{1}{V} \int \frac{i}{2}\left(\delta_{i} \hat{\Delta}_{j}^{\text {vector }}\right)_{N}(x ; x) \frac{\delta}{\delta a} \hat{\mathcal{D}}_{\text {vector }}^{i j}=3(4+7(1-\epsilon) \\
\left.\quad-5(1-\epsilon)\left(1+2 N-2 \nu_{1}\right)-(1-\epsilon)^{2}\left(N-\nu_{1}\right)\left(1+2 N-2 \nu_{1}\right)\right) a H^{2} \delta i \hat{\Delta}_{N, 1}(x ; x) \\
\frac{1}{V} \int-i\left({ }^{\alpha} \hat{\Delta}_{\text {ghost }}^{\beta}\right)_{N}(x ; x) \frac{\delta}{\delta a} \hat{\mathcal{D}}_{\alpha \beta}^{\text {ghost }}=6(1-\epsilon)\left(N-\nu_{0}\right) \\
\times\left(\left(2 \nu_{0}-2 N\right)(1-\epsilon)+\epsilon-3\right) a H^{2} \delta i \hat{\Delta}_{N, 0}(x ; x) \\
\quad+6(1-\epsilon)\left(N-\nu_{1}\right)\left(\left(2 \nu_{1}-2 N\right)(1-\epsilon)+\epsilon+1\right) a H^{2} \delta i \hat{\Delta}_{N, 1}(x ; x)
\end{aligned}
$$

The tensor contribution to (70) is

$$
-6\left(3\left(\nu_{0}-N\right)(1-\epsilon)+2\right)\left(2\left(\nu_{0}-N\right)(1-\epsilon)-3\right) a H^{2} \delta i \hat{\Delta}_{N, 0}(x ; x),
$$

while the terms in (70) multiplying $\delta i \hat{\Delta}_{N, 0}$ contribute as

$$
\begin{aligned}
& \frac{1}{1+\epsilon}\left(-4(3-\epsilon) \epsilon+(1-\epsilon)^{2}(1+3 \epsilon)\left(2 N^{2}+2 \nu_{0}^{2}\right)-(3+\epsilon)(1-\epsilon)(1-3 \epsilon)\right. \\
& \left.+N(1-\epsilon)\left(3-4 \nu_{0}-8 \epsilon\left(1+\nu_{0}\right)-3 \epsilon^{2}\left(1-4 \nu_{0}\right)\right)\right) a H^{2} \delta i \hat{\Delta}_{N, 0}(x ; x) .
\end{aligned}
$$


Finally the terms in (70) multiplying $\delta i \hat{\Delta}_{N, 2}$ contribute as

$$
\begin{aligned}
& \frac{1}{1+\epsilon}\left((1-\epsilon)^{2}(3+\epsilon)\left(2 N^{2}+2 \nu_{1}^{2}\right)+2\left(3+5 \epsilon(1-\epsilon)+\epsilon^{3}\right)\right. \\
& \left.\quad+(1-\epsilon)(3+\epsilon)\left((5+\epsilon)(1-N)-4(1-\epsilon) N \nu_{1}\right)\right) a H^{2} \delta i \hat{\Delta}_{N, 2}(x ; x)
\end{aligned}
$$

We add the corrections together to obtain the following three contributions

$$
\begin{aligned}
\frac{1}{V a^{D-1} H^{4}} \frac{\delta \Gamma_{N}^{(0)}}{\delta a}=( & -\frac{\epsilon\left(63 \epsilon^{2}+2 \epsilon-105\right)}{1+\epsilon}+\frac{1-\epsilon}{1+\epsilon}\left(3+2 N-2 \nu_{0}\right) \\
& \left.\times\left(\left(\nu_{0}-N\right)(1-\epsilon)(23+21 \epsilon)+(4+3 \epsilon)(3-7 \epsilon)\right)\right) \frac{\delta i \Delta_{N, 0}}{H^{2}} \\
\frac{1}{V a^{D-1} H^{4}} \frac{\delta \Gamma_{N}^{(1)}}{\delta a}=( & -3\left(9 \epsilon^{2}+7 \epsilon+6\right) \\
& \left.+9(1-\epsilon)\left(3+2 N-2 \nu_{1}\right)\left(\left(\nu_{1}-N\right)(1-\epsilon)-\epsilon\right)\right) \frac{\delta i \Delta_{N, 1}}{H^{2}} \\
\frac{1}{V a^{D-1} H^{4}} \frac{\delta \Gamma_{N}^{(2)}}{\delta a}=( & \frac{(5 \epsilon-6)\left(\epsilon^{2}-2 \epsilon-7\right)}{1+\epsilon} \\
& \left.-\frac{(1-\epsilon)(3+\epsilon)}{1+\epsilon}\left(3+2 N-2 \nu_{2}\right)\left(\left(\nu_{2}-N\right)(1-\epsilon)+4-\epsilon\right)\right) \frac{\delta i \Delta_{N, 2}}{H^{2}},
\end{aligned}
$$

where we have written $\delta \Gamma_{N}^{(n)}$ for the contribution to $\delta \Gamma_{N}$ that multiplies $\Delta_{N, n}$.

From these three contributions we see explicitly that indeed when $\nu=N+3 / 2$, the corrections have the correct prefactor to add up correctly to cancel the divergence in the digamma functions (85). Inserting (85) and (94) into (61) at $D=4$ we obtain the final one 
loop corrected Friedmann trace equation

$$
\begin{aligned}
& \frac{24}{\kappa}\left(\left(1-\frac{1}{2} \epsilon\right) H^{2}-\frac{1}{3} \Lambda\right)+3 p_{M}-\rho_{M}+\frac{H^{4}}{16 \pi^{2}}\left\{\beta_{1} \epsilon+\beta_{2} \epsilon^{2}+\beta_{3} \epsilon^{3}+\beta_{4} \epsilon^{4}\right. \\
& -\epsilon\left(186-149 \epsilon-11 \epsilon^{2}+10 \epsilon^{3}\right) \ln \left(\frac{(1-\epsilon)^{2} H^{2}}{4 \pi \mu^{2}}\right) \\
& +\frac{\epsilon\left(63 \epsilon^{2}+2 \epsilon-105\right)}{4(1+\epsilon)}(1-\epsilon)^{2} \\
& \times\left(\left(4 \nu_{0}^{2}-1\right)\left(\psi\left(\frac{1}{2}+\nu_{0}\right)+\psi\left(\frac{1}{2}-\nu_{0}\right)\right)-\sum_{N} \frac{64 \pi^{2}}{(H(1-\epsilon))^{2}} \delta i \Delta_{N, 0}\right) \\
& +\frac{3\left(9 \epsilon^{2}-7 \epsilon-6\right)}{4}(1-\epsilon)^{2} \\
& \times\left(\left(4 \nu_{1}^{2}-1\right)\left(\psi\left(\frac{1}{2}+\nu_{1}\right)+\psi\left(\frac{1}{2}-\nu_{1}\right)\right)-\sum_{N} \frac{64 \pi^{2}}{(H(1-\epsilon))^{2}} \delta i \Delta_{N, 1}\right) \\
& -\frac{(5 \epsilon-6)\left(\epsilon^{2}-2 \epsilon-7\right)}{4(1+\epsilon)}(1-\epsilon)^{2} \\
& \left.\times\left(\left(4 \nu_{2}^{2}-1\right)\left(\psi\left(\frac{1}{2}+\nu_{2}\right)+\psi\left(\frac{1}{2}-\nu_{2}\right)\right)-\sum_{N} \frac{64 \pi^{2}}{(H(1-\epsilon))^{2}} \delta i \Delta_{N, 2}\right)\right\} \\
& +\sum_{N}\left(3+2 N-2 \nu_{0}\right)\left(\frac{1-\epsilon}{1+\epsilon}\left(\left(\nu_{0}-N\right)(1-\epsilon)(23+21 \epsilon)+(4+3 \epsilon)(3-7 \epsilon)\right)\right) H^{2} \delta i \Delta_{N, 0} \\
& +\sum_{N}\left(3+2 N-2 \nu_{1}\right)\left(9(1-\epsilon)\left(\left(\nu_{1}-N\right)(1-\epsilon)-\epsilon\right)\right) H^{2} \delta i \Delta_{N, 1} \\
& -\sum_{N}\left(3+2 N-2 \nu_{2}\right)\left(\frac{(1-\epsilon)(3+\epsilon)}{1+\epsilon}\left(\left(\nu_{2}-N\right)(1-\epsilon)+4-\epsilon\right)\right) H^{2} \delta i \Delta_{N, 2}=0 .
\end{aligned}
$$

This equation is our final result of this section. It presents the one loop quantum corrected Friedmann trace equation, in the presence of both graviton and scalar fluctuations.

\subsection{Discussion}

Having found the one loop corrected Friedmann trace equation, we can ask the question whether the quantum effects calculated will have any significant effect. We immediately 
see from (95) that the quantum contribution is suppressed by a factor $H^{2} / m_{p}^{2}$. Because of this suppression, the one loop contribution can only become relevant for the dynamics of the Universe if there is a significant enhancement. In other words: for any significant effect the one loop correction terms should grow in time with respect to the classical tree level terms. In an accelerating universe $z_{0}$ goes to zero at late times. From (88) we see that the corrections $\delta i \Delta_{N, n}$ are proportional to $H^{4} z_{0}^{2 N+3-2 \nu_{n}}$ and thus this contribution might grow in time with respect to the background (which scales as $H^{2}$ ), making the initially tiny quantum effects significant at late times. The question whether this growth is significant is equivalent to the question whether the quantity

$$
H^{2} z_{0}^{3+2 N-2 \nu}
$$

grows in time. In an accelerating universe the fastest growing term has $N=0$. Since $z_{0}=-k \eta$ this term grows with $\eta$ as

$$
\propto \eta^{\frac{3-\epsilon}{1-\epsilon}-2 \nu}
$$

Thus we see that for the three different $\nu_{n}$ 's contributing to (95) this becomes

$$
\begin{aligned}
& \propto \eta^{\frac{3-\epsilon}{1-\epsilon}-2 \nu_{0}}=\eta^{0} \\
& \propto \eta^{\frac{3-\epsilon}{1-\epsilon}-2 \nu_{1}}=\eta^{2} \\
& \propto \eta^{\frac{3-\epsilon}{1-\epsilon}-2 \nu_{2}}=\eta^{4} .
\end{aligned}
$$

These general expressions fail near the $N=0$ pole of the digamma functions (meaning $\left.\nu_{i}=3 / 2\right)$. Here one will typically pick up an additional $\operatorname{logarithm}, \ln \left(z_{0}\right)$ from the correction terms. Thus we see that the $\nu_{0}$ contribution, in the presence of such an additional logarithm, actually grows in time. However $\nu_{0}=3 / 2$ implies $\epsilon=0$ and we see from (95) that this contribution is cancelled by the pre-factor. Thus none of the contributions actually grows in time.

The fact that the pre-factor is such that near de Sitter space the logarithmic growth drops out, is also in concordance with results obtained in [23]. This property however does not have to stay true in general. For example if higher order loop corrections are taken into account, this structure might change such that while the correction terms are extremely small, their contribution grows in time, making them significant at late enough times. Also when considering a different background geometry, one might get growing effects even at one loop order. In [16, 17] for example one loop contributions have been considered in chaotic inflationary models. Although those works found a secular growth, in [24] it was concluded that this growth disappears if one considers truly gauge invariant quantities. 


\section{Conclusion}

In this paper we have calculated the one loop contribution to the effective Friedmann equations due to graviton and matter quantum fluctuations. The background space-time we use is FLRW with the additional constraint that $\epsilon$ is constant. In such a calculation many delicate issues have to be taken into account. First of all there is mixing between the gravitational and matter degrees of freedom. This mixing can (at least on-shell) be removed by field redefinitions (31) and (40). Secondly, there is the issue of gauge freedom, which we can deal with in the standard way by adding a gauge fixing term (27) and the associated FadeevPopov ghost lagrangian (30). Finally, to calculate the one loop contribution, one needs to know the propagators. The propagators needed will however typically be infrared divergent. To regulate this infrared divergence we assumed that the spatial sections of the universe are compact. This effectively implies that the propagators should be calculated using an infrared cut-off $k_{0}$. Using this approach the scalar propagator was constructed in [1]. In the present work we have constructed the graviton and matter propagators by making use of the same method. All these technical issues can thus be resolved, leading to our final answer for the one loop Friedmann trace equation (95). Although the final answer might look intimidating, we can essentially write it as two types of contributions. One is $H^{4}$ times a constant and the other is $H^{4}$ times some power of $z_{0}=|\eta| k_{0}$. Since the tree level contribution is of the order of $H^{2} m_{p}^{2}$, we see that - as expected - quantum corrections to the Friedmann equation are suppressed as $H^{2} / m_{p}^{2}$. There are thus only two possibilities for the quantum corrections to have a significant effect. Either the coefficient in front of $H^{4}$ is large, or any of the powers of $z_{0}$ grows fast enough to compensate the suppression after a sufficient amount of time. Looking at equation (95) we see that for typical values of the parameters, the coefficient will be of order one. One might think that the digamma functions might grow large. Indeed, these functions have a simple pole when the argument is a negative integer. However, as is shown in [1], these poles are due to a logarithmic infrared divergence. These poles should therefore be subtracted by the correction terms, and in [1] it is shown that this indeed is what happens. Thus the only possibility for a significant backreaction of quantum fluctuations on the background space-time is if the correction terms grow fast enough in time. In section 4.3 we showed however that this will not happen. The only possibility where the powers of $z_{0}$ grow fast enough is the special case of de Sitter $(\epsilon=0)$. However in this case, the growing power of $z_{0}$ drops out due to the vanishing of its prefactor.

It is however not clear whether these conclusions will also hold in more general cases. Generalizations could include for example studying a more general class of backgrounds, using different regularization schemes for the infrared. For example in [45] a scheme using mode matching is introduced. This scheme gives similar results for accelerating space-times 
as the scheme used here, but the results differ for decelerating space-times. Another generalization would be to include two or higher loop contributions into account. Indeed studies in de Sitter space show, for example, that at two loop order the backreaction due to gravitons might indeed grow in time [6, 10, 8]. This paper should be seen as a first step in trying to understand backreaction on a more general background space-time.

\section{Acknowledgements}

We would like to thank Richard P. Woodard and G. Stavenga for useful discussions. The authors acknowledge financial support by Utrecht University. T.P. acknowledges financial support by FOM grant 07PR2522.

\section{References}

[1] T. M. Janssen, S. P. Miao, T. Prokopec and R. P. Woodard, "Infrared Propagator Corrections for Constant Deceleration," Class. Quant. Grav. 25 (2008) 245013 [arXiv:0808.2449 [gr-qc]].

[2] G. 't Hooft and M. J. G. Veltman, "One loop divergencies in the theory of gravitation," Annales Poincare Phys. Theor. A 20 (1974) 69.

[3] B. Allen, "The Graviton Propagator in de Sitter Space," Phys. Rev. D 34 (1986) 3670.

[4] I. Antoniadis and E. Mottola, "Graviton Fluctuations In De Sitter Space," J. Math. Phys. 32 (1991) 1037.

[5] B. Allen and M. Turyn, "An Evaluation of the Graviton Propagator in de Sitter Space," Nucl. Phys. B 292 (1987) 813.

[6] N. C. Tsamis and R. P. Woodard, "One Loop Graviton Self-Energy In A Locally De Sitter Background," Phys. Rev. D 54 (1996) 2621 [arXiv:hep-ph/9602317].

[7] N. C. Tsamis and R. P. Woodard, "The Structure of perturbative quantum gravity on a De Sitter background," Commun. Math. Phys. 162 (1994) 217.

[8] N. C. Tsamis and R. P. Woodard, "Quantum Gravity Slows Inflation," Nucl. Phys. B 474 (1996) 235 [arXiv:hep-ph/9602315].

[9] G. 't Hooft and M. J. G. Veltman, "Regularization And Renormalization Of Gauge Fields," Nucl. Phys. B 44 (1972) 189. 
[10] N. C. Tsamis and R. P. Woodard, "The quantum gravitational back-reaction on inflation," Annals Phys. 253 (1997) 1 [arXiv:hep-ph/9602316].

[11] A. Higuchi and S. S. Kouris, "On the scalar sector of the covariant graviton twopoint function in de Sitter spacetime," Class. Quant. Grav. 18 (2001) 2933 [arXiv:grqc/0011062].

[12] A. Higuchi and S. S. Kouris, "Large-distance behavior of the graviton two-point function in de Sitter spacetime," Class. Quant. Grav. 17 (2000) 3077 [arXiv:gr-qc/0004079].

[13] F. Finelli, G. Marozzi, G. P. Vacca and G. Venturi, "Adiabatic regularization of the graviton stress-energy tensor in de Sitter space-time," Phys. Rev. D 71 (2005) 023522 [arXiv:gr-qc/0407101].

[14] S. M. Christensen and M. J. Duff, "Quantizing Gravity With A Cosmological Constant," Nucl. Phys. B 170 (1980) 480.

[15] L. H. Ford, "Quantum Instability Of De Sitter Space-Time," Phys. Rev. D 31 (1985) 710.

[16] L. R. W. Abramo, R. H. Brandenberger and V. F. M. Mukhanov, "The back reaction of gravitational perturbations," arXiv:gr-qc/9702004.

[17] L. R. W. Abramo, R. H. Brandenberger and V. F. Mukhanov, "The energy-momentum tensor for cosmological perturbations," Phys. Rev. D 56 (1997) 3248 [arXiv:grqc/9704037].

[18] L. R. W. Abramo, "Energy density and pressure of long wavelength gravitational waves," Phys. Rev. D 60 (1999) 064004 [arXiv:astro-ph/9903270].

[19] V. F. Mukhanov, L. R. W. Abramo and R. H. Brandenberger, "On the back reaction problem for gravitational perturbations," Phys. Rev. Lett. 78 (1997) 1624 [arXiv:grqc/9609026].

[20] B. Losic and W. G. Unruh, "On leading order gravitational backreactions in de Sitter spacetime," Phys. Rev. D 74 (2006) 023511 [arXiv:gr-qc/0604122].

[21] G. Cognola, E. Elizalde, S. Nojiri, S. D. Odintsov and S. Zerbini, "One-loop $f(R)$ gravity in de Sitter universe," JCAP 0502 (2005) 010 [arXiv:hep-th/0501096].

[22] T. Janssen and T. Prokopec, "A graviton propagator for inflation," Class. Quant. Grav. 25 (2008) 055007 arXiv:0707.3919 [gr-qc]. 
[23] L. R. W. Abramo and R. P. Woodard, "One loop back reaction on power law inflation," Phys. Rev. D 60 (1999) 044011 [arXiv:astro-ph/9811431].

[24] L. R. Abramo and R. P. Woodard, "No one loop back-reaction in chaotic inflation," Phys. Rev. D 65 (2002) 063515 [arXiv:astro-ph/0109272].

[25] L. R. Abramo and R. P. Woodard, "Back-reaction is for real," Phys. Rev. D 65 (2002) 063516 [arXiv:astro-ph/0109273].

[26] J. Iliopoulos, T. N. Tomaras, N. C. Tsamis and R. P. Woodard, "Perturbative quantum gravity and Newton's law on a flat Robertson-Walker background," Nucl. Phys. B 534 (1998) 419 [arXiv:gr-qc/9801028].

[27] T. Janssen, S.P. Miao and T. Prokopec, "Graviton one-loop effective action and inflationary dynamics," arXiv:0807.0439.

[28] N. C. Tsamis and R. P. Woodard, "Dimensionally regulated graviton 1-point function in de Sitter," Annals Phys. 321 (2006) 875 [arXiv:gr-qc/0506056].

[29] L. Parker, "Quantized fields and particle creation in expanding universes. 1," Phys. Rev. 183 (1969) 1057.

[30] T. M. Janssen, S. P. Miao, T. Prokopec and R. P. Woodard, "The Hubble Effective Potential," arXiv:0904.1151 [gr-qc].

[31] A. Bilandzic and T. Prokopec, "Quantum radiative corrections to slow-roll inflation," Phys. Rev. D 76 (2007) 103507 [arXiv:0704.1905 [astro-ph]].

[32] M. S. Sloth, "On the one loop corrections to inflation and the CMB anisotropies," Nucl. Phys. B 748 (2006) 149 [arXiv:astro-ph/0604488].

[33] M. S. Sloth, "On the one loop corrections to inflation. II: The consistency relation," Nucl. Phys. B 775 (2007) 78 [arXiv:hep-th/0612138].

[34] R. H. Brandenberger, "Lectures on the theory of cosmological perturbations," Lect. Notes Phys. 646 (2004) 127 [arXiv:hep-th/0306071].

[35] V. F. Mukhanov, H. A. Feldman and R. H. Brandenberger, "Theory of cosmological perturbations. Part 1. Classical perturbations. Part 2. Quantum theory of perturbations. Part 3. Extensions," Phys. Rept. 215 (1992) 203. 
[36] L. H. Ford and L. Parker, "Infrared Divergences In A Class Of Robertson-Walker Universes," Phys. Rev. D 16 (1977) 245.

[37] A. Vilenkin and L. H. Ford, "Gravitational Effects Upon Cosmological Phase Transitions," Phys. Rev. D 26 (1982) 1231.

[38] A. D. Linde, "Scalar Field Fluctuations In Expanding Universe And The New Inflationary Universe Scenario," Phys. Lett. B 116 (1982) 335.

[39] S. Nobbenhuis, "Categorizing different approaches to the cosmological constant problem," Found. Phys. 36 (2006) 613 [arXiv:gr-qc/0411093].

[40] B. Allen, "Vacuum States In De Sitter Space," Phys. Rev. D 32 (1985) 3136.

[41] E. Mottola, "Particle Creation In De Sitter Space," Phys. Rev. D 31 (1985) 754.

[42] N. A. Chernikov and E. A. Tagirov, "Quantum theory of scalar fields in de Sitter spacetime," Annales Poincare Phys. Theor. A 9 (1968) 109.

[43] S. P. Miao and R. P. Woodard, "The fermion self-energy during inflation," Class. Quant. Grav. 23, 1721 (2006) [arXiv:gr-qc/0511140];

S. P. Miao, "The quantum-corrected fermion mode function during inflation," arXiv:0705.0767 [hep-th].

[44] E. O. Kahya and R. P. Woodard, "Quantum Gravity Corrections to the One Loop Scalar Self-Mass during Inflation," Phys. Rev. D 76 (2007) 124005 [arXiv:0709.0536 [gr-qc]].

[45] T. Janssen and T. Prokopec, "Regulating the infrared by mode matching: A massless scalar in expanding spaces with constant deceleration," [arXiv:0906.0666 [gr-qc]].

[46] N. D. Birrell and P. C. W. Davies, "Quantum Fields In Curved Space," Cambridge, Uk: Univ. Pr. (1982) $340 p$

[47] A. O. Barvinsky, A. Y. Kamenshchik and I. P. Karmazin, "The Renormalization Group For Nonrenormalizable Theories: Einstein Gravity With A Scalar Field," Phys. Rev. D 48 (1993) 3677 [arXiv:gr-qc/9302007].

[48] R. E. Kallosh, O. V. Tarasov and I. V. Tyutin, Nucl. Phys. B 137 (1978) 145.

[49] R. P. Woodard, "Quantum effects during inflation," arXiv:astro-ph/0310757.

[50] R. P. Woodard, "de Sitter breaking in field theory," arXiv:gr-qc/0408002. 